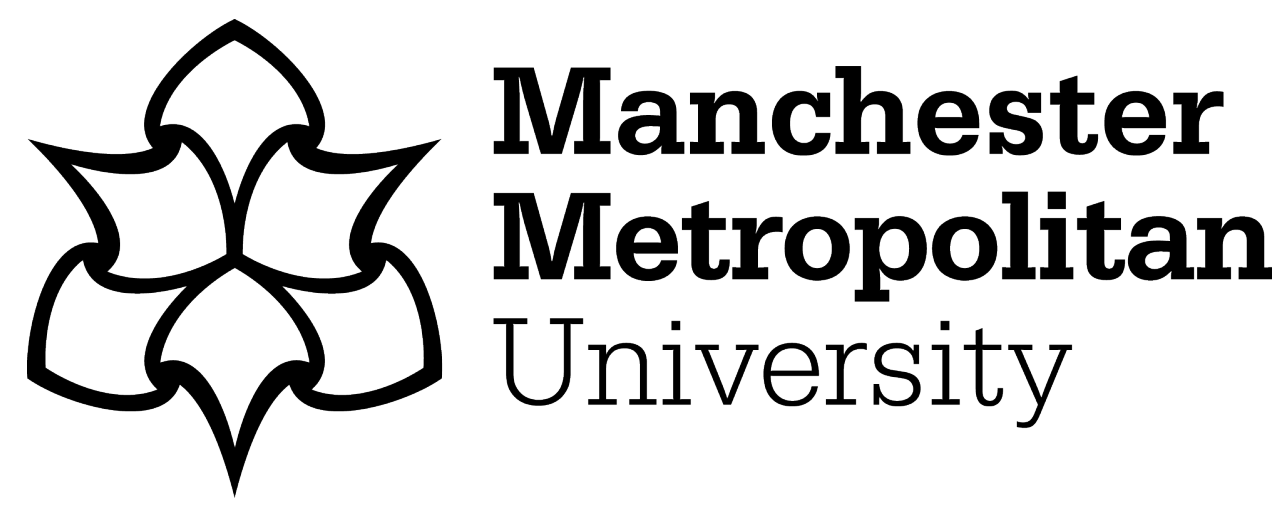

Valitov, G, Jamshidi, R ORCID logoORCID: https://orcid.org/0000-00018407-8005, Rossi, D, Gavriilidis, A and Mazzei, L (2019) Effect of acoustic streaming on continuous flow sonocrystallization in millifluidic channels. Chemical Engineering Journal, 379. ISSN 1385-8947

Downloaded from: https://e-space.mmu.ac.uk/625161/

Version: Accepted Version

Publisher: Elsevier

DOI: https://doi.org/10.1016/j.cej.2019.122221

Please cite the published version 


\title{
Effect of Acoustic Streaming on Continuous Flow Sonocrystallization in Millifluidic Channels
}

\author{
Gleb Valitov, Rashid Jamshidi, Damiano Rossi, Asterios Gavriilidis, Luca Mazzei* \\ University College London, Department of Chemical Engineering, Torrington Place, London WC1E TJE, UK \\ *Corresponding author: I.mazzei@ucl.ac.uk
}

\begin{abstract}
This work is concerned with the effect of ultrasound on mixing and consequently on crystallization in millifluidic channels. An ultrasonic horn with frequency of $20 \mathrm{kHz}$ was placed inside a vessel with a well-defined geometry containing a single capillary tube with internal diameter (I.D.) of 1.55 or 3.2 $\mathrm{mm}$ operated with a fluid flow rate between 1 and $17.6 \mathrm{ml} / \mathrm{min}$. This system was employed to produce crystals of adipic acid in the range of 15-35 $\mu \mathrm{m}$. The effect of ultrasound on flow patterns, residence time distribution (RTD) and cooling crystallization were investigated experimentally and numerically. Simulations of acoustic and velocity fields inside the millichannel acting as crystallizer allowed characterizing the acoustic streaming generated within it. In the large capillary, especially at small flow rates, acoustic streaming influenced the flow field, inducing vortices and leading to significant changes in RTD. Conversely, in the small capillary, ultrasound affected the flow field and the RTD negligibly, and a laminar velocity profile with straight streamlines was obtained. As a consequence, different crystallization behaviours were observed in the two capillaries; in particular, while the mean crystal size increased with the sonication residence time in the $1.55 \mathrm{~mm}$ I.D. capillary, it decreased in the $3.2 \mathrm{~mm}$ I.D. capillary. This difference highlights the importance of considering acoustic streaming when designing sonocrystallizers.
\end{abstract}

\section{Introduction}

Crystallization is an established process for purification of compounds in a mixture based on their solubility. The conditions at which a crystallizer is operated can affect the size and shape of crystals, the resulting yield and the type of polymorphous form obtained, which, in turn, affect the degree of crystallinity of the end product and influence its physical and chemical stability. Furthermore, the bioavailability of a pharmaceutical compound is also affected by the morphology and crystal size distribution (CSD). To achieve the required product quality, industrial manufacturers introduce additional processing steps, thus increasing the cost of production. Currently, crystalline products 
are manufactured in batch mode using standard stirred tank reactors, with seeding as a means of controlling the crystallization process. Nevertheless, the nonuniform spatial distribution of process parameters, such as temperature and concentration, leads to heterogeneous conditions in stirred tank crystallizers. This, in turn, has a negative impact on the CSD. A number of post-crystallization processes, such as milling or granulation, are currently employed to reduce the CSD width. Being able to develop crystallization processes that are controllable and can lead to narrow CSDs is therefore one of the main challenges for the pharmaceutical industry.

The bulk of current engineering research on crystallization focuses on developing alternatives to the industrial practice by using established intensification and process control techniques. Moving from batch to continuous operations presents the benefit of having time-independent temperature and concentration profiles, which leads to products with more stable and reproducible characteristics and avoids the issue of inconsistent batch-to-batch output [1,2]. Microfluidic devices offer enhanced mass and heat transfer owing to high surface-to-volume ratio. Comparatively uniform temperature and concentration distributions, as well as enhanced control and reproducibility, are widely reported in the literature on continuous microfluidic devices $[3,4]$. However, in a continuous crystallizer issues arise due to encrustation and accumulation of crystals over time, which is particularly problematic at the microscale $[5,6]$.

Ultrasound is a process intensification tool that may significantly improve the crystallization process in terms of controllability, product quality and production rate [7]. Application of ultrasound in the chemical industry is widespread in fields like sonochemistry, sonoemulsification, ultrasonic cleaning and others $[8,9]$. In chemical syntheses, ultrasound has been commonly utilized to accelerate reaction kinetics and even develop new synthetic pathways [10]. In the context of crystallization, the use of ultrasound is referred to as sonocrystallization, and ultrasound has been proven to positively influence the kinetics of the various processes involved in the crystal production (in particular the nucleation process), which has several potential benefits [11-14]:

- The enhancement of nucleation kinetics can render seeding unnecessary, thereby eliminating a processing step [2].

- The enhancement of nucleation kinetics leads to the reduction of the metastable zone width; this permits reducing operational costs (e.g. cooling load, antisolvent consumption etc.) and shortening induction times (time, at a given supersaturation, after which crystals become detectable) $[2,15]$.

- The enhancement of growth kinetics leads to faster growth of crystals, which allows reducing the volume of the crystallizer required to obtain crystals of a given mean size [7]. 
1 Continuous sonocrystallization has been investigated by several research groups. Their work has clearly revealed the potential of this technology, but also the complexity of the process. Ultrasound has been used both to enhance crystallization kinetics in Mixed Suspension Mixed Product Removal (MSMPR) crystallizers [16] and, more commonly, as a method for generating seeds in tubular crystallizers $[4,17,18]$. For the latter, ultrasound has been employed to induce nucleation at lower supersaturation levels (but, usually, nuclei are grown in the absence of ultrasound). Such approach is common when in-situ generation of seeds is required; however, the main focus of these studies was the downstream process for the investigation of crystal growth after the sononucleation stage $[4,12,17,19]$. For example, Eder et al. [19] combined ultrasound with other techniques, like Taylor flow and crystal healing (dissolution of smaller crystals to narrow the CSD), to enhance crystal size control; Siddique et al. [20] used sonication to generate seeds which were fed to oscillatory baffled reactors. Since in these studies the focus was not on ultrasound, the generated seeds were characterized experimentally, and an investigation of the acoustic field was not conducted. In other studies, the sonicated regions were carefully designed to understand and improve the seeding process $[21,22]$. In these works, the effects of ultrasound on nucleation temperature, crystal morphology and/or crystallization yield were of interest, and greater control over the acoustic field was achieved. Investigating the effects of cavitation on crystallization was the goal of these efforts. Until now, the effects of acoustic streaming on crystallization in continuous-flow systems have been overlooked in the literature. This is the focus of the present work.

The propagation of sound waves in a liquid medium generates an oscillatory pressure field which affects the fluid via two mechanisms: cavitation and acoustic streaming. Cavitation can originate when a pressure wave passing through a liquid creates voids that may collapse in the compression phase of the pressure oscillation. This, however, requires that the pressure amplitude exceed the tensile strength of the pure liquid, which is the lowest negative pressure the liquid can sustain without homogeneous nucleation of a cavity. For water, this value is suggested to be $-100 \mathrm{MPa}$ [23] or $-27 \mathrm{MPa}$ [24] according to theory and measurements, respectively. These values are well below the negative acoustic pressure ( $a$ few bars) that can be reached in the system employed in this work. Cavitation bubbles can also originate from existing gas pockets (bubble nuclei), stabilized in the liquid or trapped in the crevices on the tube walls; this is referred to as gas bubble cavitation. The cavitation activity in our system is essentially due to the latter. The oscillatory pressure field acts on the bubble nuclei present in the liquid; the bubbles grow and shrink during the negative and positive pressure periods, respectively. Two phenomena can occur as a result of bubble volume variation: stable cavitation and inertial/transient cavitation. In the former, the bubble volume increases and decreases with the acoustic pressure in a periodic fashion, and the minimum and maximum values 
of the bubble volume are nearly constant, the pulsation being cyclical. In the latter, the extreme values of the bubble volume are not constant; the bubble grows in size over time, until reaching a critical volume at which it implodes. When this happens, the fluid within the bubble briefly reaches extreme conditions (up to $5000 \mathrm{~K}$ and 1000 bar for a time interval of about 0.01 to 0.1 nanoseconds) [25-27] and releases a powerful shock wave. During the collapse of a bubble, the flow field around it is affected; this phenomenon is referred to as microjetting.

Viscous attenuation of an acoustic wave travelling though the fluid results in a time-averaged motion in the fluid that is called acoustic streaming [28]. Note that this motion is different from the Stokes drift caused by the sound wave propagation and the microjets in the fluid generated by the shock waves occurring during inertial bubble collapse [29]. The streaming flows can manifest in two types: boundary layer driven streaming (vortices are formed near the boundaries of the fluid domain) and Eckart streaming (vortices are formed in the bulk flow). The former prevails in milli- and microfluidic devices only at high frequencies ( 1 to $5 \mathrm{MHz}$ ), because it arises due to high energy dissipation in the boundary layer of a fluid along solid boundaries; such energy dissipation does not occur in the system investigated in this study, since the wavelength is much larger than the thickness of the boundary layer. The exact threshold value for the channel size at which boundary layer streaming is observed must be determined based on frequency and fluid properties [30]. It is commonly accepted that if the characteristic length of the channel is larger than a quarter of the wavelength then boundary layer streaming can occur. In general, for the frequencies used in sonocrystallization and sonochemistry (up to $200 \mathrm{kHz}$ ), the channel dimensions should be of the order of $10 \mathrm{~cm}$ or higher for boundary layer streaming to be significant. In the present study, at a frequency of $20 \mathrm{kHz}$, a quarter of the wavelength in water is $\sim 2 \mathrm{~cm}$. This is an order of magnitude larger than the diameter of the largest channel considered $(3.2 \mathrm{~mm})$; therefore, boundary layer streaming is not expected to occur. On the other hand, Eckart streaming arises owing to dissipation of energy in the bulk of the fluid. The loss of acoustic energy results in a steady momentum flux that forms streaming of fluid along the direction of the sound wave [31]. In the majority of conventional applications, the momentum flux induced by ultrasound affects negligibly the (already existing) fluid velocity field, unless the sound pressure amplitude is unconventionally high and the fluid moves slowly. On the other hand, in lab-scale setups where the flow characteristic length is small $(\mathrm{mm}$ to $\mathrm{cm})$, the streaming created by ultrasound can play a role in altering the flow field. Various numerical and experimental studies were conducted to investigate the effect of acoustic streaming on the flow in milli-scale setups. For example, Wiklund et al. [31] demonstrated experimentally that Eckart streaming vortices can have a representative velocity scale larger than that characterizing the velocity of the bulk flow. They 
the capillary sizes in our study. Other studies investigated and characterized acoustic streaming of all origins (boundary layer as well as Eckart streaming); these are summarised in various recent reviews on the topic $[30,32,33]$. Nonetheless, owing to the geometry-specific behaviour that fluids have in the presence of ultrasound, these studies hold little relevance to our work.

This work focuses on investigating the effects of acoustic streaming on the hydrodynamic behaviour of a milliscale crystallizer and is divided into four sections. Section 2 presents the experimental system and outlines the methodology adopted to characterize sonocrystallization and measure the residence time distribution in the channels. Section 3 shows the modelling approaches we adopted to characterize the ultrasound wave, flow field (including acoustic streaming) and RTDs. Section 4 discusses the results of the numerical model. Section 5 presents experimental results which show the effect of ultrasound, and specifically of acoustic streaming, on mixing and crystallization; these experimental results are also used to validate the predictions of the model.

\section{Experimental Section}

\subsection{Materials}

Adipic acid (hexanedioic acid, $\left(\mathrm{CH}_{2}\right)_{4}(\mathrm{COOH})_{2},>99.5 \%$ pure, Sigma-Aldrich, UK) was the crystallizing substance selected for this research work. It was used as received without further purification. The adipic acid solution was made using deionized water (conductivity $<0.2 \mu \mathrm{S} / \mathrm{cm}$ ) as solvent. The solid density of adipic acid at $20^{\circ} \mathrm{C}$ is $1.36 \mathrm{~g} / \mathrm{cm}^{3}$. The solubility of adipic acid in water at various temperatures was determined from the solubility curve reported [34]. Adipic acid was also selected due to the absence of polymorphism, which simplifies the analysis of the impact of ultrasound on crystallization.

\subsection{Experimental System and Procedure}

\subsubsection{Millifluidic Sonocrystallization Experiments}

Figure 1 shows the schematic of the capillary sonocrystallizer adopted in this work. The feed solution was prepared by dissolving a controlled amount of adipic acid in distilled water to achieve a concentration of $2.12 \mathrm{~g}$ of AA per $100 \mathrm{~g}$ of water; this is the saturation concentration at room temperature $\left(22^{\circ} \mathrm{C}\right)$. Dissolution took place at $60^{\circ} \mathrm{C}$ while the mixture was mixed with a magnetic stirrer. After all crystals were dissolved, the feed vessel was removed from the hotplate and kept at room temperature for the duration of the experiments. The feed solution temperature was always above $22{ }^{\circ} \mathrm{C}$, ensuring that the solution was undersaturated and no nuclei were present in the feed vessel. A syringe pump (Mitos Duo XS-Pump, Dolomite, UK) was used to pump the adipic acid solution from the feed vessel into the pre-cooling section (Figure 2A), where the solution was cooled 
1 to $10^{\circ} \mathrm{C}$ (at this temperature, the solubility of adipic acid is $1 \mathrm{~g}$ of AA per $100 \mathrm{~g}$ of water) to reach a

2

3

4 supersaturation ratio of 2.12 (this is defined as the ratio of the solute concentration to the solubility at the temperature considered). The pre-cooling section was a two-pass heat exchanger which consisted of a $4 \mathrm{~cm} \times 4 \mathrm{~cm} \times 20 \mathrm{~cm}$ Perspex chamber in which the cooling fluid flowed, a coiled capillary tube made of FEP (fluorinated ethylenepropylene, $1.55 \mathrm{~mm}$ or $3.2 \mathrm{~mm}$ internal diameter, $0.8 \mathrm{~mm}$ wall thickness, BOLA) in which the adipic acid solution flowed, and an annular "tube holder" (a 3D printed structure which directed the flow of cooling water and held the capillary tube). The cooling water was pumped using a cooling water recirculator (Huber, Ministat 125) at the flow rate of 6-7 $\mathrm{I} / \mathrm{min}$, which was sufficient to ensure that the cooling water temperature did not change throughout the system. The time that the supersaturated solution spent in the pre-cooling section was minimized by changing the length of capillary tube in this section to avoid formation of nuclei prior to sonication while still ensuring that the desired outlet temperature of $10{ }^{\circ} \mathrm{C}$ was reached. The precooling section was connected to the sonication section by a PTFE nut and ferrules; the distance between the two was kept to a minimum to reduce heat transfer with the environment.

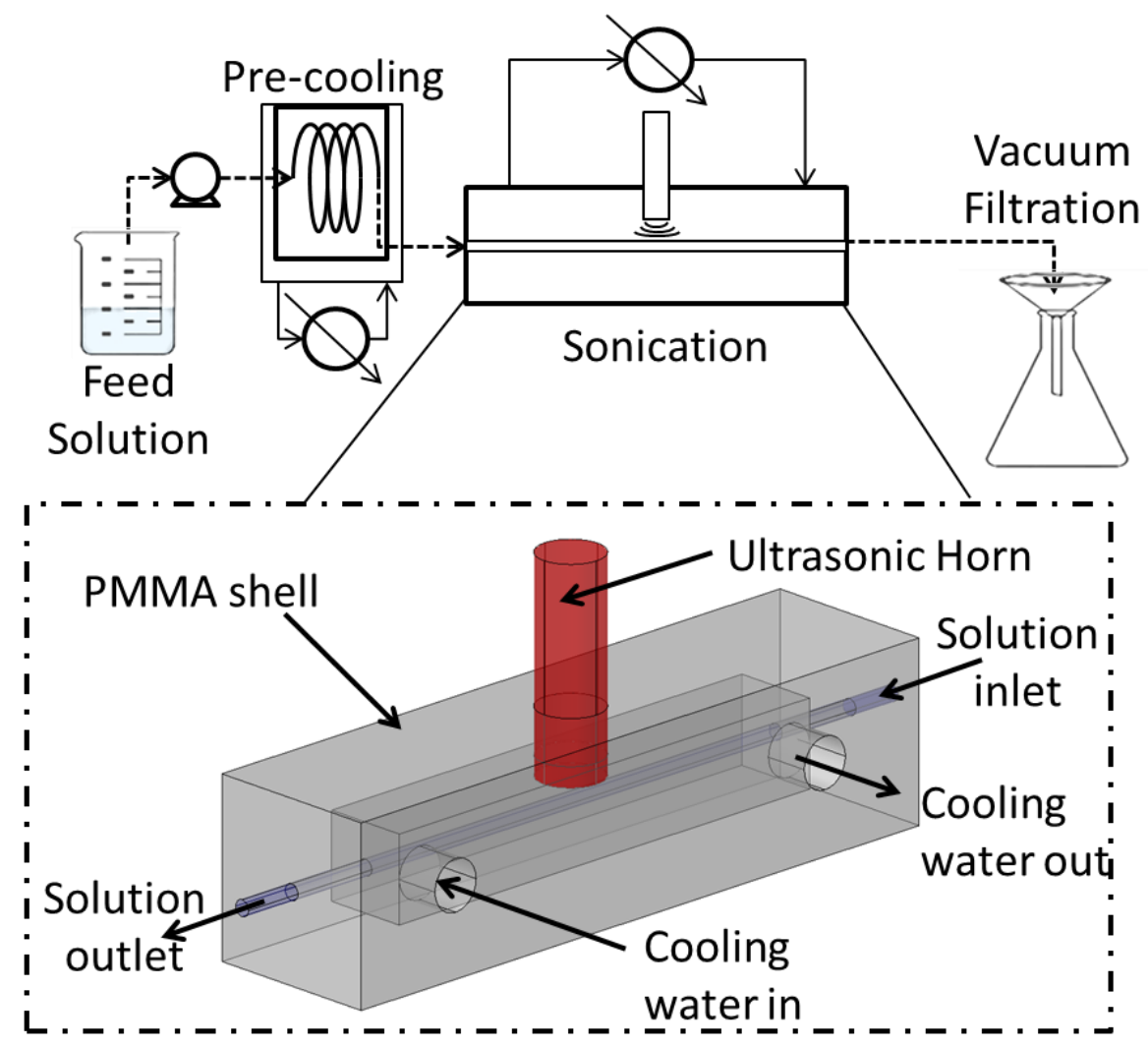

Figure 1. Schematic of the capillary sonocrystallizer setup. 

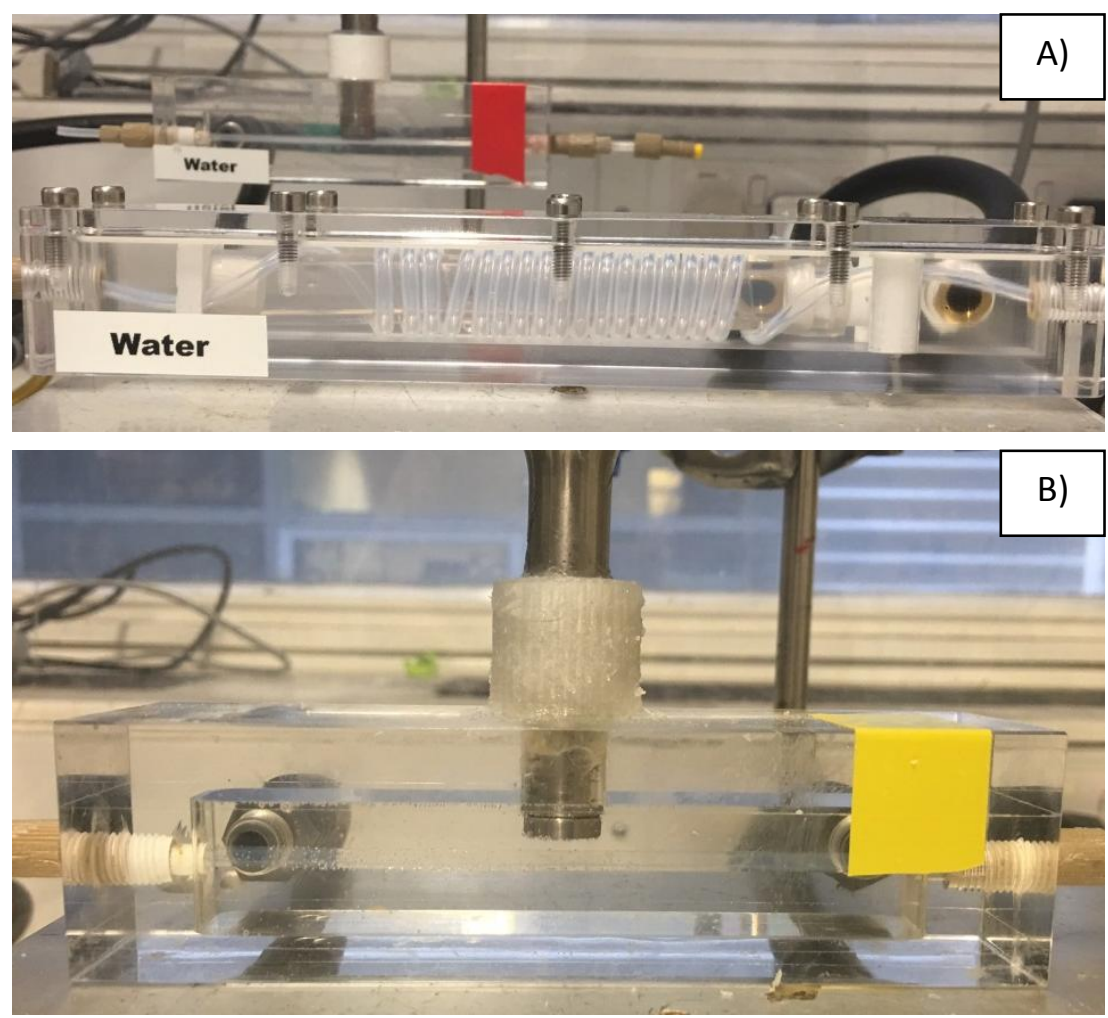

Figure 2. Pictures of $A$ ) pre-cooling section and B) sonication section

The sonication section (Figure $2 \mathrm{~B}$ ) consisted of a straight capillary tube placed horizontally inside a large chamber in which cooling water flowed. This chamber acted as cooling jacket, counteracting the heating produced by ultrasound (owing to dissipation of acoustic energy). The solution was maintained at constant temperature along the capillary. An ultrasound horn (Cole-Parmer, $750 \mathrm{~W}$ Ultrasonic Processor with $1 / 2$ in. flat tip, frequency $20 \mathrm{kHz}$, amplitude $21 \%$ ) was placed inside the chamber containing the cooling water at a controlled distance away from the capillary. This reduced the heating effect from the ultrasonic source. To keep the capillary horizontal, two threads were made in the centre of the container lateral walls, and the capillary was tightened between two opposite male nuts. To verify that the temperature inside the capillary was constant, a thin thermocouple probe was inserted into the capillary from the outlet and moved upstream until the tip of the thermocouple probe was located under the centre of the ultrasonic horn. The temperature was measured using deionised water before the crystallization experiments were conducted (the thermocouple was removed before the solution of adipic acid was introduced into the system). We found that heating due to sound energy dissipation within the liquid was counterbalanced by the heat taken away by the cooling water, so that the liquid inside the capillary tube remained at a constant temperature of $10 \pm 0.2^{\circ} \mathrm{C}$. The sonoprobe was aligned with the capillary (see Figures 1 and 2) and the distance between them was kept constant. For both capillary tubes used, the distance between the centreline of the capillary and the sonoprobe was kept at $2.8 \mathrm{~mm}( \pm 0.05 \mathrm{~mm})$ 
in order to ensure that the acoustic pressure distributions were similar in the two capillary tubes. A horn with frequency of $20 \mathrm{kHz}$ was selected to ensure presence of cavitation activity, while still enabling to investigate acoustic streaming. Even if at higher frequencies acoustic streaming is more significant, increasing the frequency to values with far more pronounced streaming effects would result in a considerable reduction (and possible entire loss) of the effect of ultrasound on nucleation (via cavitation), because the cavitation activity decreases as the frequency of ultrasound increases. This is why frequencies above $200 \mathrm{kHz}$ are rarely used for applications in which cavitation plays a crucial role, as in sonocrystallizers. For this reason, we did not consider wave frequencies higher than $20 \mathrm{kHz}$.

Crystals were filtered as soon as they left the sonication section using a $0.3 \mu \mathrm{m}$ Whatman cellulose nitrate membrane. The membrane was dried for $10 \mathrm{~min}$ on a hot plate at $60^{\circ} \mathrm{C}$ and weighed to determine the mass of the crystals collected. After $10 \mathrm{~min}$, we observed that the filter paper weight did not change with time, which indicates that all water had evaporated. The following equation was employed to calculate the yield of the process:

$$
Y \equiv \frac{\Delta M_{\text {filter }}}{\left(C_{\text {in }}-C^{*}\right) * \dot{V} * t_{\text {collection }}} * 100 \% \text { (eq. 2.1) }
$$

where $Y$ represents the percentage of the mass of adipic acid crystallized, $\Delta M_{f i l t e r}$ is the mass of adipic acid filtered, $C_{i n}$ is the inlet concentration of adipic acid, $C^{*}$ is the saturation concentration of adipic acid at the temperature inside the crystallizer, $\dot{V}$ is the volumetric flow rate and $t_{\text {collection }}$ is the collection time. The crystal yield $Y$ was determined over a $0.5-5$ min collection time.

The crystals were subsequently suspended in vegetable oil - in which adipic acid is insoluble - and homogeneously dispersed using a low power ultrasonic water bath for $35 \mathrm{~min}$. The power of the ultrasonic bath was $50 \mathrm{~W}$. However, only a fraction of this power reached the sample, due to the low volume of the sampling vial (5-10 $\mathrm{ml})$ containing the vegetable oil/crystal suspension compared to the volume (2 I) of the ultrasonic bath. Cavitation was not expected in the vegetable oil owing to the low bubble nuclei volume fraction (air is insoluble in oil) and the high cavitation threshold in vegetable oil (owing to high surface tension and viscosity). This slurry was used to determine the crystal size distribution with a Beckman Coulter LS13320 single wavelength laser diffraction particle size analyser. To check the reproducibility of the experiments, each single experiment was repeated 6-18 times. At each repeat, one sample was collected; subsequently the system was cleaned with deionized water to ensure no crystals remained in the capillary before the next repeat. The yield for each sample was measured once and the CSD for each sample was measured three times and averaged. The mean diameter $D_{4,3}$ (defined as the ratio of the fourth to the third moments of the 
CSD) was calculated from each distribution and regarded as representative of the mean crystal size for that distribution. For each case considered, we report the average value of all the repeats and the error bar (plus/minus one standard deviation of the experimental values about the average; this is not to be confused with the standard deviation of the crystal size distribution).

\subsubsection{Residence Time Distribution Measurements}

As outlined in the introduction, mixing owing to acoustic streaming can occur in the presence of ultrasound, thus affecting the crystallization process. In order to characterize the extent of mixing, the residence time distribution (RTD) of the sonication section was measured. The precooling section was removed during the RTD measurements, because only the mixing under the sonoprobe was of interest, given that crystallization occurred there. $100 \mathrm{ml} \mathrm{SGE}$ glass syringes were filled with carrier fluid (deionized water) and tracer fluid (aqueous solution of Basic Blue 3, Dye content 25\%, Sigma-Aldrich). A solution of adipic acid in water has similar physical properties to deionized water and so there was no need to use adipic acid solutions for RTD measurements. It was assumed that crystals have negligible effect on the RTD on account of their small size and low volume fraction. Based on the crystal yield values, the volume fraction of crystals in the solution was estimated to be between 0.001 and 0.005 . Therefore, the fluid-crystal suspensions were extremely dilute. Thus, direct interactions between particles (collisions and/or frictional contacts) were unlikely to be present. Moreover, the size of the crystals and possible aggregates was small enough so that they followed the streamlines of the fluid. This can be verified by calculating the Stokes number for the crystals and the aggregates. This number is defined as the ratio between the relaxation time of the particles (the time that the particles require to reach local dynamical equilibrium) and the time scale of the flow. The Stokes number of individual crystals (with a typical size of $20 \mu \mathrm{m}$ ) had an order of magnitude of $10^{-3}$. The largest clusters of crystals we observed under the microscope were of about $100 \mu \mathrm{m}$, for which the Stokes number was still well below $10^{-1}$. Therefore, we could assume that crystals and aggregates relax rapidly in the flow and follow the streamlines without altering the flow field of the continuous phase.

The syringes were mounted on a dual syringe rack attached to a pump (Harvard Apparatus PHD 2000). The two fluids were simultaneously pumped to a six-way injection HPLC valve (M-461, IDEX) that allowed switching between the tracer and the carrier fluid flowing within the tube. One fluid was disposed of as waste, while the other was fed to the capillary tube in the sonication section. Initially the carrier fluid flowed through the channel. At a time $t_{0}$, the feed was abruptly switched to tracer fluid, generating a step change in tracer concentration. At the tube outlet, an optical fibre, connected to a light source (Ocean Optics DH-2000-BAL), shone light through the capillary tube, 
which passed through a flow cell (see schematic in the Supplementary Information). The light that passed through the fluid reached, via optical fibres, a spectrophotometer (Ocean Optics USB2000+UV-Vis-ES) whereby the light intensity was measured at small regular time intervals (10-25 ms). The RTD curve was then obtained by analysing the absorbance data according to the equation:

$$
E(t)=\frac{d}{d t}\left[\frac{C_{\text {tracer }}(t)}{C_{\text {tracer }, 0}}\right]=\frac{d}{d t}\left[\frac{A(t)}{A_{\max }}\right]
$$

where $E$ is the RTD curve, $C_{\text {tracer }}$ is the concentration of tracer (Blue Dye), $C_{\text {tracer }, 0}$ is the initial concentration of tracer and $A$ is the absorbance. It must be noted that equation 2.2 only applies when the concentration is directly proportional to the absrobance, which was proven to be the case for this dye and capillary in our prior work [27].

\section{Theory}

\subsection{Acoustic Streaming Modelling}

The propagation of small amplitude pressure waves in a fluid was described by the homogeneous wave equation [35]:

$$
\frac{\partial^{2} p(\boldsymbol{x}, t)}{\partial t^{2}}-c^{2} \nabla^{2} p(\boldsymbol{x}, t)=0
$$

where $p$ is the acoustic pressure, $c$ is the speed of sound in the fluid, $\boldsymbol{x}$ is the spatial position vector and $t$ is the time. If one assumes that the acoustic wave is a sinusoidal function of time, determining the field $p(\boldsymbol{x}, t)$ reduces to calculating the spatial distribution of the amplitude of the pressure wave, which we denote as $P(\boldsymbol{x})$. This is governed by the following partial differential equation, referred to as frequency-domain wave equation [36]:

$$
\nabla^{2} P(x)+k^{2} P(x)=0(e q .3 .2)
$$

Here $k \equiv \omega / c$ is the wavenumber and $\omega$ is the angular frequency of the wave. Conventionally, one would solve this equation for the liquid phase. In the presence of cavitation bubbles, the ultrasound wave attenuation is significant and must be accounted for. To this end, we considered the linear model suggested by Commander and Prosperetti [37], where a complex wavenumber $k_{m}$ replaces $k$ :

$$
k_{m}^{2}=\left(\frac{\omega}{c}\right)^{2}\left(1+\frac{4 \pi c^{2} n_{b} R_{0}}{\omega_{0}^{2}-\omega^{2}+2 i \beta \omega}\right)(\text { eq. 3.3) }
$$

Here $R_{0}$ is the size of the initial bubble nuclei (assumed to be monodispersed air bubbles) present in the liquid, $\beta$ is the damping coefficient of the wave, $\omega_{0}$ is the bubble resonance frequency and $n_{b}$ is 
1 the bubble number density (i.e., number of bubbles per unit volume). Measuring $R_{0}$ and $n_{b}$ is 2 difficult; in contrast, the volume fraction of bubbles $\phi$ (i.e., volume of bubbles per unit volume of solution) is a measurable quantity, equal to (4/3) $\pi R_{0}^{3} n_{b}$. Selecting a typical value of $3 \times 10^{-4}$ for $\phi$ [37] and taking $R_{0}=30 \mu \mathrm{m}$ [38], the corresponding value of $n_{b}$ is $2.65 \times 10^{9} \mathrm{~m}^{-3}$. The bubble population was assumed to be uniformly distributed in space. These bubbles dampen the acoustic wave owing to viscous, thermal and acoustic phenomena. This effect was accounted for via the damping coefficient $\beta$, which is given by:

$$
\beta \equiv \frac{2 \mu}{\rho R_{0}^{2}}+\frac{p_{0}}{2 \rho \omega R_{0}^{2}} \mathfrak{I}(\Phi)+\frac{\omega^{2} R_{0}}{2 c} \quad(e q .3 .4)
$$

where $\mu$ and $\rho$ are the viscosity and density of the fluid, respectively, and $p_{0}$ is the undisturbed fluid pressure in the bubble position, which is higher than the equilibrium pressure in the fluid $\left(p_{\infty}\right)$ by an amount of $2 \sigma / R_{0}$ due to the action of the surface tension $\sigma$. The complex dimensionless parameter $\Phi$, whose imaginary part appears in the above equation, is defined as:

$$
\Phi \equiv \frac{3 \gamma}{1-3(\gamma-1) i \chi\left[\left(\frac{i}{\chi}\right)^{0.5} \operatorname{coth}\left(\frac{i}{\chi}\right)^{0.5}-1\right]}(\text { eq. 3.5) }
$$

where $\gamma$ is the specific heat ratio of the gas (air) inside the bubble, $\chi \equiv \alpha / \omega R_{0}^{2}$ and $\alpha$ is the thermal diffusivity of the gas. The resonance frequency of the bubbles is given by:

$$
\omega_{0}^{2} \equiv \frac{p_{0}}{\rho R_{0}^{2}}\left[\Re(\Phi)-\frac{2 \sigma}{R_{0} p_{0}}\right]
$$

where $\mathfrak{R}(\Phi)$ is the real part of the complex number $\Phi$.

The imaginary part of the complex wave number $k_{m}$ is called the attenuation coefficient and is a measure of the absorption of the ultrasound energy in the liquid. The energy absorption, which is the cause of acoustic streaming, is considered via a force term in the linear momentum balance equation of the fluid. Thus, in the presence of ultrasound and in steady-state conditions, this balance equation takes the following form:

$$
\boldsymbol{u} \cdot \nabla \boldsymbol{u}-v \nabla^{2} \boldsymbol{u}+\frac{\nabla p_{h}}{\rho}=\boldsymbol{F}(\text { eq. 3.7) }
$$

where $v$ is the kinematic viscosity of the fluid, $\boldsymbol{u}$ is its velocity and $p_{h}$ is the hydrodynamic pressure (which is related to the fluid motion and is different from the acoustic pressure $p$ generated by ultrasound). We simulated one extreme case (where acoustic streaming was the strongest: $3.2 \mathrm{~mm}$ 
1 I.D. capillary, sonication time of the fluid beneath the horn of $3 \mathrm{~s}$ ) employing a turbulent flow model $2 \quad(k-\varepsilon)$, and we confirmed that the laminar linear momentum balance equation (Eq. 3.7) was valid for the system investigated and the effect of turbulence was negligible (the turbulent viscosity value did not exceed $1 \%$ of the fluid molecular viscosity). The quantity $\boldsymbol{F}$ is the source term that accounts for acoustic streaming and is referred to as acoustic radiation force (here it has the units of $N / \mathrm{m}^{3}$, because the linear momentum balance equation is written per unit volume for an incompressible fluid). This force is given by [39]:

$$
F=\frac{\left|\Im\left(k_{m}\right)\right|}{\rho c^{2}} \sum_{j=1}^{\infty} j^{2} P_{j}^{2} \quad \text { (eq. 3.8) }
$$

in which $P_{j}$ is the amplitude of the $j$-th harmonic of the acoustic wave. Usually, one can assume that the first harmonic of the wave $(j=1)$ has higher amplitude than all the other harmonics. This is a reasonable assumption when the boundaries of the wave-propagating medium are made of acoustically absorbing materials and do not strongly reflect acoustic waves. In our system, the boundaries are made of PMMA (cooling jacket walls) and FEP (capillary). Hence, with reasonable accuracy, we considered only the first harmonic of the wave to calculate the acoustic radiation force. The amplitude of the first harmonic can be obtained by solving Eq. 3.2, which provides a complex scalar field whose real part represents the acoustic pressure amplitude. For further details about this point, we refer to the Supplementary Information (and the references therein cited).

When solving Eq. 3.2, we considered cooling water to be stationary. This assumption does not lead to inaccurate predictions of the acoustic pressure field. Cavitation and acoustic streaming do occur also in the cooling water. We accounted for the former in the wave propagation equation when evaluating the acoustic pressure field (Eqs. 3.3-3.6), because the cavitation bubbles contribute to the damping of the sound wave. Acoustic streaming, conversely, was not considered in the acoustic pressure field calculations, since the fluid motion affect neither the physical properties of water nor the propagation of the sound wave.

Additionally, the thickness of the capillary wall $(0.75 \mathrm{~mm})$ is significantly lower than the wavelength of the ultrasound wave $(7.5 \mathrm{~cm})$; hence, the effect of the capillary on wave propagation can be neglected. This is because, over the thickness of the capillary wall, the ultrasound wave does not change significantly. Moreover, the material properties (density and speed of sound) of the capillary wall that affect wave propagation are similar to those of water. Hence, effects such as reflection from the capillary surface and scattering of the wave can be neglected. In light of these considerations, in the simulation of the acoustic pressure the capillary wall was not considered. Also 
the material properties inside and outside the capillary are almost identical (the inner solution is water with $2.12 \mathrm{~g} \mathrm{AA} / 100 \mathrm{~g}$ and the outer fluid is cooling deionized water); thus, for the acoustic pressure field simulations, we considered a single domain consisting of cooling water.

The numerical simulations were performed via the Finite Element Method (FEM) using COMSOL Multiphysics 5.3a. The acoustic pressure field in the channel was calculated using the frequencydomain wave equation, which we solved using the following boundary conditions:

- At the wall of the cooling jacket, we used a Dirichlet boundary condition: $P=0$.

- On the side walls of the horn, we used a Neumann boundary condition: $\partial_{n} P=0$, where $\mathrm{n}$ denotes the direction normal to the solid surface.

- At the surface of the tip of the horn: $P=\sqrt{2 \rho c P o / A_{\text {tip }}}$, where $A_{\text {tip }}$ is the surface area of the tip and $P o$ is the ultrasound source power; the latter was measured, with a calorimetric method, in a prior study we conducted, in which the same horn was used [42].

- Because the capillary tube is not considered in the acoustic pressure field calculations, no boundary conditions on its walls were required.

The simulations for the acoustic pressure field were conducted in steady state and the computation time on a computer with Intel Xeon $3.5 \mathrm{GHz}$ CPU and $192 \mathrm{~GB}$ of RAM was around one hour for 757,494 Degrees of Freedom in the finite element model. The physical properties of the fluid used for the numerical simulations are reported in Table 1.

Table 1: Physical properties of the fluid and constants used for the acoustic field simulations.

\begin{tabular}{ccccccc}
\hline$\gamma$ & $\alpha\left(\mathrm{m}^{2} / \mathrm{s}\right)$ & $\sigma(\mathrm{N} / \mathrm{m})$ & $\rho\left(\mathrm{kg} / \mathrm{m}^{3}\right)$ & $\mu(\mathrm{Pa} \cdot \mathrm{s})$ & $c(\mathrm{~m} / \mathrm{s})$ & $P o(W)$ \\
\hline 1.4 & $1.9 \times 10^{-5}$ & 0.0725 & 999.7 & $1.3 \times 10^{-3}$ & 1498 & 8.3 \\
\hline
\end{tabular}

\subsection{Residence Time Distribution Modelling}

We used numerical simulations to solve the mass balance equation for the fluid and the set of eqs. 3.2 and 3.7. Our solution strategy was as follows: by knowing the acoustic boundary conditions on the boundaries of the computational domain, we first solved eq. 3.2 to find the amplitude of the acoustic pressure at each point in space. We used this field to calculate the acoustic radiation force (eq. 3.8). Thereafter, by knowing the hydrodynamic boundary conditions on the boundaries of the domain, we solved the set of mass $[\nabla \cdot(\rho \boldsymbol{u})=0$ ] and linear momentum balance (eq. 3.7) equations for the fluid. This yielded the velocity and hydrodynamic pressure fields, which can be used to study the mixing and residence time distributions in the capillary tube in the presence of ultrasound. 
Upon calculating the fluid velocity field inside the capillary, one may investigate how acoustic streaming affects the crystallization process. The kinetics and mechanisms of crystallization in the presence of ultrasound are not well understood in the literature. However, there is no debate that the solute concentration in the solution has a significant role on the resulting yield and crystal size. Due to difficulty of directly measuring the concentration in the system, we looked at the residence time distribution in the capillary tube and drew conclusions about the effectiveness of mixing and in turn of changes in solute concentration. If acoustic streaming caused a significant change in the RTD, then the solute concentration field also changed, thereby altering the output of the crystallizer.

We obtained RTDs both numerically and experimentally. The experimental RTDs accounted for the mixing induced by both acoustic streaming and microjetting (or by microjetting alone in the cases where acoustic streaming was negligible). To study the relative importance of the two processes, we determined the RTDs numerically as well, with a model that accounted solely for acoustic streaming. Now, in the cases where acoustic streaming is negligible, if the numerical and experimental RTDs are quite similar, one can conclude that mixing due to microjetting is unimportant. Similarly, in the cases where acoustic streaming is significant, if the two RTDs are close to one another, one can conclude that mixing is essentially caused solely by acoustic streaming. Therefore, by relating mixing to crystallization, one can also relate crystallization to acoustic streaming. To determine the RTDs numerically, we solved the mass balance equation for a single, non-reactive species. This can be written as:

$$
\frac{\partial C}{\partial t}=-\boldsymbol{u} \cdot \nabla C+D_{m} \nabla \cdot \nabla C(\text { eq.3.9) }
$$

where $C$ is the concentration, $D_{m}$ is the molecular diffusion coefficient (assumed to be constant) and $\boldsymbol{u}$ is the velocity field, which is calculated via eq. 3.7.

The mass conservation equation was solved numerically (in a time dependent simulation), with a boundary condition at the channel inlet which represents a step change in tracer concentration:

$$
C=0 \text { for } t<0 ; C=C_{\text {inlet }} \text { for } t \geq 0
$$

At the walls of the channel, we specified a zero-flux boundary condition; at the outlet of the channel, the diffusive flux normal to the boundary was set to zero. Note that, for the cases studied in this work, the Peclet number $\left(P e=L \bar{u} / D_{m}\right)$ was significantly larger than unity (of order $10^{4}$ ); consequently, the influence of the outlet boundary condition was noticeable only in close proximity to the outlet boundary and was minimal in the rest of the domain [40]; we verified that this was the case numerically. The tube in the simulations was $0.1 \mathrm{~mm}$ longer than that in the experiments and 
1 the outlet concentration used to determine the RTD was taken $0.1 \mathrm{~mm}$ from the outlet of the

2

3

4

$$
\begin{gathered}
F(t)=\frac{C(t)}{C_{\max }}(\text { eq. 3.10) } \\
E(t)=\frac{d F}{d t}(\text { eq. 3.11) }
\end{gathered}
$$

$9 C_{\max }$ being the maximum concentration at the channel outlet (its value is equal to that of $C_{\text {inlet }}$, which is specified in the inlet boundary condition).

Time-dependent simulations of the RTD were conducted on the same computer as the one used for the acoustic pressure field calculations. There was a total of 19,462,691 Degrees of Freedom; the convergence criterion for the relative error for the residuals was set to $10^{-3}$ and the computational time was two weeks per simulation.

For ease of comparison of simulation results with experimental results and literature models, the resulting $E$ curves were made dimensionless:

$$
\begin{gathered}
\widehat{E}(\theta) \equiv E(t) * \tau_{R} \quad(\text { eq. 3.12) } \\
\theta \equiv \frac{t}{\tau_{R}} \quad(\text { eq.3.13) }
\end{gathered}
$$

where $\tau_{R}$ is the mean residence time, equal to the first moment of the RTD function:

$$
\tau_{R} \equiv \int_{0}^{\infty} t E(t) d t \quad(e q .3 .14)
$$
[41], which is given by:

$$
\hat{E}(\theta)=\frac{1}{2 \theta^{3}} \text { for } \theta>0.5(\text { eq. } 3.15)
$$




\section{4. Results}

\section{$2 \quad 4.1$ Modelling and Experiments for the Sonocrystallizer Design}

3

Figure 3 shows the cross-sectional distribution of the acoustic pressure field inside the sonication section of the crystallizer. Our prior work suggested that, under the assumptions made here, transient cavitation occurs when the acoustic pressure amplitude exceeds 2 bar [43]. Based on this information, we estimated the region inside the capillary tube where the acoustic pressure is sufficiently high to induce transient cavitation. It was vital to consider the domain in which transient cavitation occurs, since this is a key mechanism in the induction of nucleation [43]. The experiments were conducted at a supersaturation level at which, without ultrasound, the induction time (the time required by crystals to become visible) was of the order of $10 \mathrm{~min}$ (in this case, the crystals formed and grew at the wall due to heterogeneous nucleation), while, in the presence of ultrasound, it was less than $1 \mathrm{~s}$ (nucleation occurred in the bulk of the fluid, owing to the presence of transient cavitation; crystals became detectable within a second after ultrasound was turned on). This value was characteristic for all the cases investigated, with and without acoustic streaming. The time scale of acoustic streaming was of the order of $0.1 \mathrm{~s}$ (this is given by the ratio between the length scale and the velocity scale of the vortexes created by ultrasound), thus being one order of magnitude smaller than that of the induction time. Hence, within the first second after ultrasound was turned on, both acoustic streaming and crystallization became significant. To ensure that acoustic streaming had time to develop and that the initial nuclei (formed immediately after ultrasound was applied) did not affect the results, the system was allowed to reach steady-state conditions. In the results presented in Sections 4.4 and 4.5, all crystals were collected between 2 and 7 min after turning on the ultrasound: $2 \mathrm{~min}$ was sufficient for the system to reach steady state, while $7 \mathrm{~min}$ was insufficient for heterogeneous nucleation at the walls to play any significant role. Note that, as previously mentioned, before running each single experiment, we cleaned the system with deionized water, which ensured that initially no crystals were present, in particular at the walls of the capillary. Consequently, in the regions where transient cavitation was absent, it was reasonable to assume that nucleation was negligible. By identifying the region where transient cavitation occurred, we also established the region where ultrasound had a significant effect on the kinetics of nucleation. 


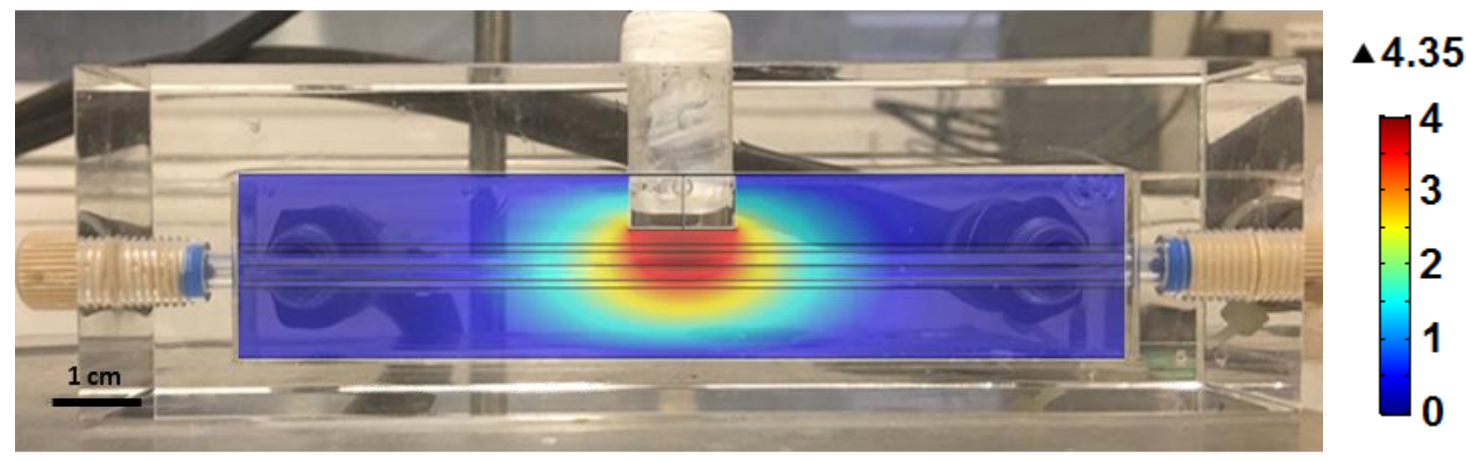

1

Figure 3: Acoustic pressure amplitude (in bar) at the vertical cross-section inside the cooling jacket of the crystallizer. The acoustic pressure map is overlaid on the picture of the experimental system to better represent the location of the capillary tube where crystallization occurs.

From Figure 3, we can see that the acoustic pressure amplitude drops below 2 bar at a distance of about $4.5 \mathrm{~mm}$ from the horn tip. Thus, no transient cavitation is expected to occur at larger distances from the tip of the horn. In order to ensure nearly uniform acoustic conditions over the vertical cross-section of the crystallizer beneath the horn tip, we needed to ensure that no cavitation-free zones were present within this region. This provided an upper bound for the capillary tube diameter: the bottom inner wall of the tube could not be further than $4.5 \mathrm{~mm}$ away from the tip of the horn. Experimentally, we also determined that the upper outer wall of the capillary should not be closer than $0.5 \mathrm{~mm}$ to the tip of the horn. If it was closer, the cooling water did not fully circulate around the capillary and heating due to ultrasound started to pose an issue. Hence, taking into account the thickness of the capillary wall $(0.75 \mathrm{~mm})$, we concluded that the largest commercially available capillary that we could use had to have has an internal diameter (I.D.) of 3.2 $\mathrm{mm}$.

The lower bound for the capillary size was selected based on clogging considerations. Experiments were conducted to find the smallest capillary tube size for which clogging did not occur. The results showed that channels with I.D. of $1 \mathrm{~mm}$ (or less) frequently blocked, regardless of the supersaturation and flow rate used. The time at which the capillary tube clogged was random, from a few seconds up to several minutes. Opposite results were obtained when the capillary tube internal diameter was larger. Capillary tubes with 1.55, 2.40 and $3.20 \mathrm{~mm}$ I.D. did not present any clogging issues for the selected range of flow rates $(0.5$ to $17.6 \mathrm{ml} / \mathrm{min})$. In all these cases, crystals were instantly produced in the sonication section (the section of the tube beneath the sonoprobe). Thus, the lower bound for the capillary size was identified to be $1.55 \mathrm{~mm}$ I.D. For the above reasons, 
1 studies were conducted for both $1.55 \mathrm{~mm}$ and $3.2 \mathrm{~mm}$ I.D. capillary tubes to compare the behaviour 2 at the extremes.

3

4

\subsection{Effect of Acoustic Streaming on the Flow Field}

In order to investigate the effects of acoustic streaming and cavitation, we altered the exposure to ultrasound by varying the mean residence time of the solution under the horn $\left(\tau_{S}\right)$. This time was controlled by the solution flow rate. In general, when the flow rate was changed, both sonication residence time and extent of mixing owing to acoustic streaming were affected. The diameter of the capillary was also a factor that influenced acoustic streaming. By carefully selecting flow rate and tube size, we varied the sonication residence time and/or the influence of acoustic streaming on the flow patterns. The feasible range for the sonication residence time is reported below and was dictated by experimental limitations and by the need to consider equal values of $\tau_{S}$ in the two capillaries investigated (so that their performance could be compared).

1. The upper bound for $\tau_{S}$ was selected to be $\sim 3 \mathrm{~s}$, which corresponds to a flow rate of 0.5 $\mathrm{ml} / \mathrm{min}$ in the $1.55 \mathrm{~mm}$ I.D. capillary. At lower flow rates, the $1.55 \mathrm{~mm}$ I.D. capillary tended to clog; hence, reducing the flow rate further was unfeasible.

2. The lower bound for $\tau_{S}$ was selected to be $\sim 0.3 \mathrm{~s}$, which corresponds to a flow rate of 17.6 $\mathrm{ml} / \mathrm{min}$ in the $3.2 \mathrm{~mm}$ I.D. capillary. At higher flow rates, issues arose in crystal collection: the filters flooded, and crystals started growing on the filter paper. Consequently, increasing the flow rate further was not possible with the experimental set-up employed. 
In these two extremes, the mean sonication residence time differs by an order of magnitude, which is sufficient for the analysis that we intended to carry out. An additional point was selected between the two extremes; thus, three mean sonication residence times, proposed to establish the behaviour of the crystallizer, were considered: $3.0,1.0$ and $0.3 \mathrm{~s}$. The corresponding flow rates were 2.0 $\mathrm{ml} / \mathrm{min}, 6.9 \mathrm{ml} / \mathrm{min}$ and $17.6 \mathrm{ml} / \mathrm{min}$ for the $3.2 \mathrm{~mm} \mathrm{I.D.} \mathrm{capillary} \mathrm{tube} \mathrm{and} 0.5 \mathrm{ml} / \mathrm{min}, 1.5 \mathrm{ml} / \mathrm{min}$ and $4.8 \mathrm{ml} / \mathrm{min}$ for the $1.55 \mathrm{~mm}$ I.D. capillary tube.

$1.55 \mathrm{~mm}$ Tube I.D.

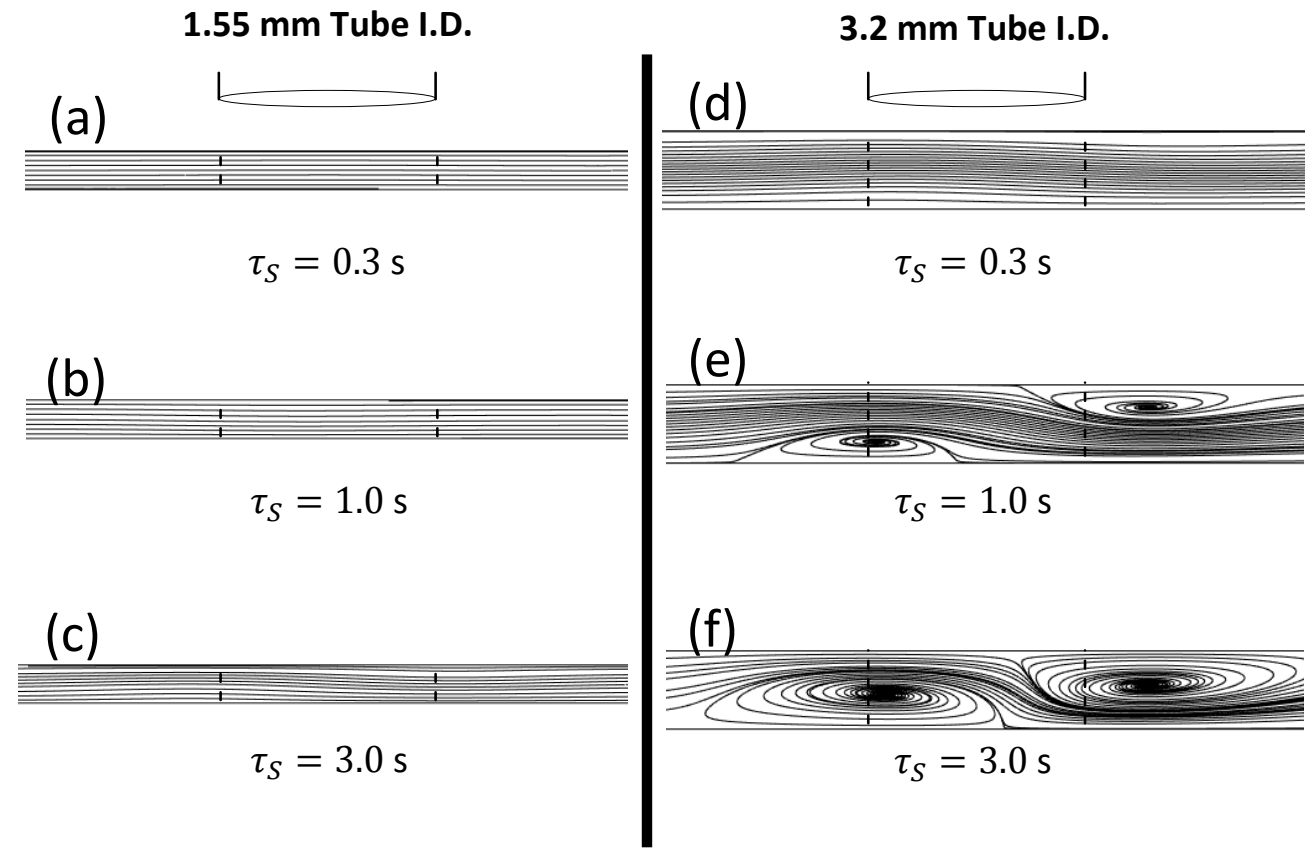

Figure 4: Streamlines inside the capillary tube at different mean sonication residence times for the $1.5 \mathrm{~mm} I D$ capillary at flow rates of (a) $4.8 \mathrm{ml} / \mathrm{min}$, (b) $1.5 \mathrm{ml} / \mathrm{min}$ and (c) $0.5 \mathrm{ml} / \mathrm{min}$ and for the 3.2 $\mathrm{mm} I D$ capillary at flow rates of (d) $17.6 \mathrm{ml} / \mathrm{min}$, (e) $6.7 \mathrm{ml} / \mathrm{min}$ and (f) $2 \mathrm{ml} / \mathrm{min}$. Dotted lines show the location of the ultrasonic horn. The magnitude of the velocity field for each case is available in the Supplementary Information.

7 Using the acoustic pressure field in Figure 3, and solving equations 3.7 for the capillary tube, one can calculate the velocity profile inside the tube. The resulting flow patterns for the selected capillary sizes and mean residence times are displayed in Figure 4. In the capillary tube with $1.55 \mathrm{~mm}$ I.D., acoustic streaming has negligible effect on the flow pattern. In this case, a laminar flow velocity profile with straight streamlines is obtained for all residence times, although the streamlines are slightly bent at the largest residence time considered (however, the deviation is negligible). In the $3.2 \mathrm{~mm}$ I.D. capillary tube, acoustic streaming affects significantly the flow pattern. As observed, at the largest flow rate considered, which results in a mean sonication residence time of $0.3 \mathrm{~s}$, the flow pattern resembles that of a laminar flow in silent conditions; the deviation is minor. Therefore, for this residence time, the laminar flow velocity profile with straight streamlines assumption is valid. For $\tau_{S}=1.0 \mathrm{~s}$, we observe two small vortices inside the capillary tube beneath the horn (later on 
referred to as underdeveloped vortices), each covering less than half of the tube vertical crosssection. These lead to local regions of back-mixing; however, the bulk of the flow does not experience enhanced mixing and the streamlines have a serpentine profile. For $\tau_{S}=3.0 \mathrm{~s}$, the vortices take up most of the sonication region and acoustic streaming has a dominant effect on the fluid velocity field. In the latter case, the vortices could even be observed by naked eye during crystallization experiments and RTD measurements. In capillary tubes of intermediate size between $1.55 \mathrm{~mm}$ I.D. and $3.2 \mathrm{~mm}$ I.D., fully developed vortices were not observed via numerical simulations. For instance, in a $2.4 \mathrm{~mm}$ I.D. capillary tube, for the same range of sonication residence times, vortices either did not form or were underdeveloped (being similar to those observed in the $3.2 \mathrm{~mm}$ I.D. capillary tube for $\left.\tau_{S}=1.0 \mathrm{~s}\right)$. In this capillary, fully developed vortices did not develop. Thus, as said, within the limitations of the experimental system adopted, the $3.2 \mathrm{~mm}$ I.D. capillary tube was the tube of smallest size in which vortices could take up most of the sonicated region. In light of this, we chose not to consider tubes of intermediate size (between $1.55 \mathrm{~mm}$ I.D. and $3.2 \mathrm{~mm}$ I.D.), because in them the full extent of the effect of acoustic streaming on crystallization could not be investigated.

Overall, the $1.55 \mathrm{~mm}$ I.D. capillary can be considered as a reference for studying the effect of acoustic streaming on crystallization. In this capillary, in the selected fluid velocity range, acoustic streaming is negligible; hence, one can study the effects of sonication residence time alone. On the other hand, the larger (3.2 mm I.D.) capillary can be used to investigate the combined effect of sonication residence time and acoustic streaming on crystallization. By comparing the two cases, one can acquire insight into the extent to which acoustic streaming influences the performance of the crystallizer.

\subsection{Effect of Acoustic Streaming on Residence Time Distribution}

The main goal of this work is to outline the effect of acoustic streaming on crystallization; both are complex processes, and there is no evidence for a direct relationship between them. It is well known that crystallization kinetics depend on solute concentration. The solute concentration spatial profile is strongly affected by mixing, and since this in turn is affected by acoustic streaming, this process is expected to play an important role in the crystal synthesis. One can characterize how acoustic streaming affects mixing by determining the residence time distribution of the system. Numerically, there are two ways to evaluate the RTD from a known velocity field: Lagrangian Particle Tracking (LPT) or solving the mass balance equation for the tracer (i.e., equation 3.9). The former method assumes massless particles in the system whose deterministic velocity is equal to the fluid velocity and whose stochastic velocity results in a random walk related to the tracer diffusivity in the fluid. 
1 One can consider neglecting the effect of diffusion when the time scale of diffusion $\left(\tau_{D}=d^{2} / 4 D\right.$, where $d$ is the tube diameter and $D$ is the tracer diffusivity) is orders of magnitude larger than the average residence time in the capillary $\left(\tau_{R}=L / u\right.$, where $L$ is the tube length and $u$ is the mean fluid velocity). This can often be done for laminar flows in capillaries in the absence of ultrasound, where the streamlines are straight and the RTD can be estimated via LPT with no random walk (in this case, our numerical results are in agreement with experimental data and with the theoretical "convection model" [41]). However, in the presence of eddies induced by acoustic streaming, the laminar flow with straight streamlines assumption no longer applies. The diameter of the capillary can no longer be considered as characteristic length in the diffusion time scale; this instead coincides with the size characterizing the smallest eddies generated by acoustic streaming, which can be much smaller than the diameter of the capillary [44]. Consequently, the diffusion time becomes much smaller and mixing via diffusion can no longer be neglected. Integrating the acoustic model and the LPT model with random walk is complex, computationally expensive and time consuming. Hence, we opted for the other available approach: solving the mass balance equation for the tracer (that is, equation 3.9). The underlying disadvantage of this methodology is the error introduced by numerical diffusion. Liu [45] developed a methodology for estimating this error. By carefully designing the mesh for the simulated domain, we could reduce the resulting numerical error to less than $9 \%$ of the diffusion term.

Without ultrasound, for both capillary sizes and all flow rates, the streamlines were straight and the flow had the Poiseuille velocity profile. So, the dimensionless RTDs for all cases were the same. The theoretical model which applies to such flow is the convection model (details of this model are in Section 3.2) and the dimensionless RTD from this model can be found in Figure 5. It was found that without ultrasound the normalized root-mean-square deviation (NRMSD) between the RTD of the theoretical pure convection model and that obtained numerically was only 0.003 , while the NRMSD between the latter and the experimental RTD was only 0.005; the match, therefore, was satisfactory. The NRMSD adopted here is defined as:

$$
N R M S D=\frac{1}{\hat{E}_{r}(\theta)_{\max }-\hat{E}_{r}(\theta)_{\min }} \sqrt{\frac{\sum_{i=1}^{N}\left[\hat{E}_{r}\left(\theta_{i}\right)-\widehat{E}_{m}\left(\theta_{i}\right)\right]^{2}}{N}}
$$

where $\hat{E}_{r}(\theta)$ is the RTD from the numerical model or experiments and $\hat{E}_{m}(\theta)$ is the RTD from the analytical model described by equation 3.15 .

As outlined in the introduction, there are two forms of streaming: boundary layer streaming and Eckart streaming; furthermore, the collapses of cavitation bubbles generate microjetting. All these 
1 processes may affect mass transfer and, therefore, the evolution of the tracer concentration profile and the RTD of the system. However, boundary layer streaming was assumed to be negligible owing to the acoustically transparent nature of the boundary material (FEP) and to the low wave frequency $(20 \mathrm{kHz})$ considered in this study (boundary layer streaming prevails in millifluidic devices at higher frequencies, above $1 \mathrm{MHz}$ ). Conversely, the effects of both Eckart streaming and microjetting may be important in characterizing mixing within the system. We showed that the contribution of microjetting was negligible. To do so, we considered a scenario in which the Eckart streaming is negligible (this is the case in Figure 4 for the $1.55 \mathrm{~mm}$ I.D. capillary with a mean sonication residence time $\tau_{S}$ of $0.3 \mathrm{~s}$; as we see, the fluid streamlines are essentially straight). In this case, consequently, only microjetting, induced by cavitation bubbles, affects mixing. Figure 5 shows the results of the experiments as well as of the simulations and of the theoretical convection model. The last two are essentially identical. This was expected because the numerical simulations account only for acoustic streaming (which in this case is negligible) and not for microjetting, whilst the theoretical model neglects both. More importantly, one can observe that the difference between experimental results and theory, albeit present, is quite small (the NRMSD is equal to 0.008). This suggests that the effect of microjetting on the RTD is indeed negligible. For the $1.55 \mathrm{~mm}$ I.D. capillary, the dimensionless RTDs for all sonication residence times looked similar to the RTD reported in Figure 5. In all cases, for this capillary size, acoustic streaming was negligible and the RTD closely resembled that of the Pure Convection Model, which holds in the absence of ultrasound (the values of the NRMSDs between convention model, experimental data and numerical results never exceeded 0.01). 


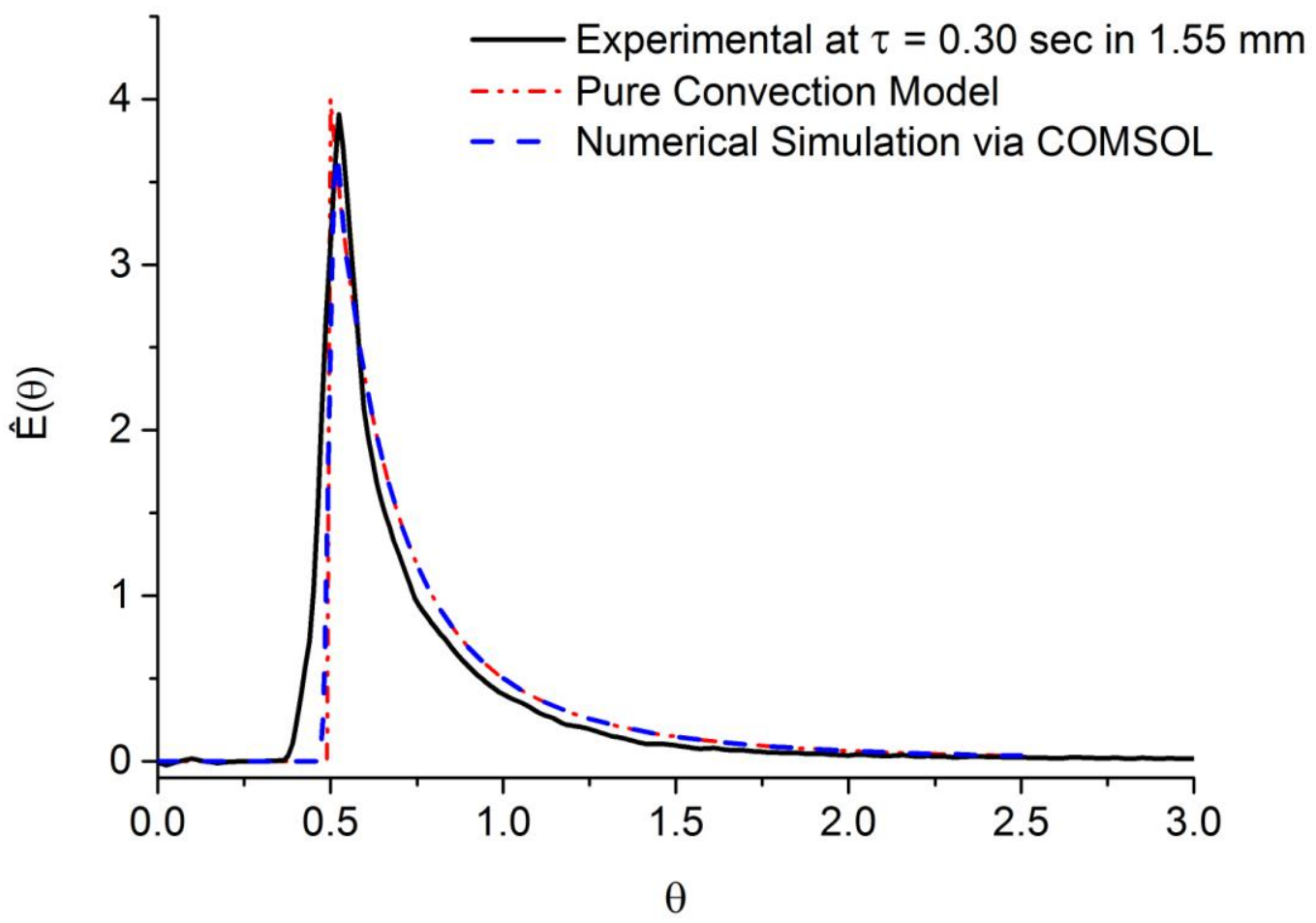

Figure 5: Residence Time Distribution in the presence of ultrasound-induced cavitation and without significant Eckart acoustic streaming effects (sonication residence time of $0.30 \mathrm{~s}$ in the $1.55 \mathrm{~mm}$ I.D. capillary).

Next, we considered the other extreme case, where Eckart acoustic streaming changes significantly the fluid velocity field; this was clearly observed in the $3.2 \mathrm{~mm}$ I.D. capillary at $\tau_{S}=3.0 \mathrm{~s}$ (Figure 4). The resulting RTD is presented in Figure 6. Note that the results of the theoretical convection model are not presented because, due to the non-negligible effect of acoustic streaming, which distorts the streamlines and generates vortices, the model is not applicable. The experimental results show the effect of Eckart acoustic streaming; this is reasonably similar to that revealed by the numerical simulations (the NRMSD is equal to 0.067). The shape of the RTD curve for this case is close to that of the dispersion flow model, which indicates the presence of mixing in the system with diffusion no longer being negligible. Also, the multiple peaks present in the numerical results might be an indicator of internal recirculation [41]. In the experimental RTD, the small secondary peaks are absent, possibly owing to the difficulty of detecting such anomalies in the F-curve, where small fluctuations in the derivative are usually lost when one removes the instrument noise and differentiates the F-curve to produce the E-curve (i.e. the RTD graph presented here). The change in 
1 streaming affects significantly the solute concentration profile under the horn, and in turn the 2 kinetics of nucleation and crystal growth.

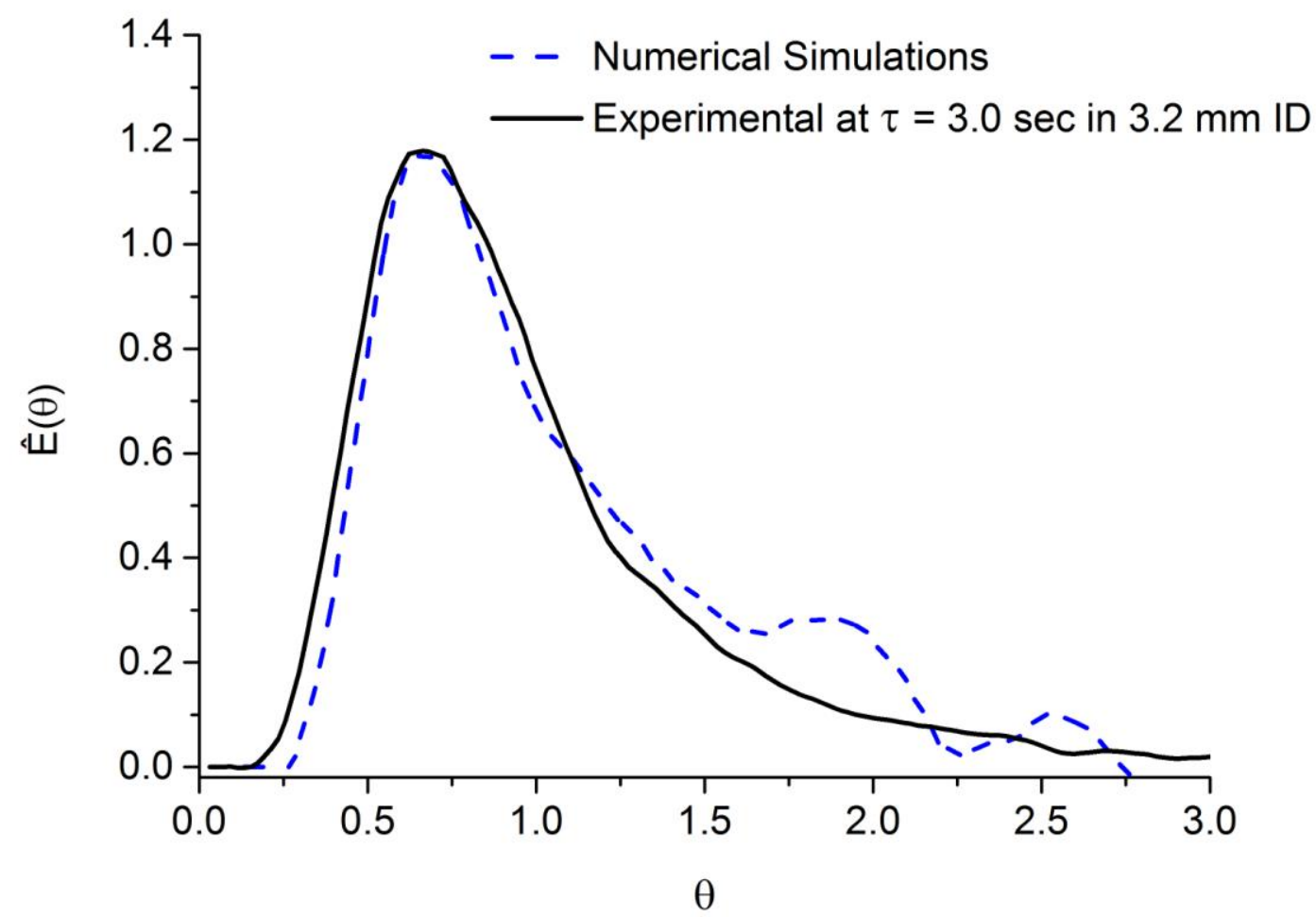

3

Figure 6: Residence Time Distribution in the presence of ultrasound-induced cavitation with significant Eckart acoustic streaming effects (sonication residence time of $3.0 \mathrm{~s}$ in the $3.2 \mathrm{~mm}$ I.D. capillary).

Figures 7 and 8 show the RTDs for the $3.2 \mathrm{~mm}$ capillary at mean sonication residence times of 0.3 and $1.0 \mathrm{~s}$, respectively. Based on the results in Figure 7, one can confirm that the minor changes to the laminar velocity profile induced by the Eckart acoustic streaming do not have a significant effect on the RTD. Both the numerical and experimental results closely fit the predictions of the theoretical convection model. Thus, Eckart streaming has negligible effect (as does microjetting). 


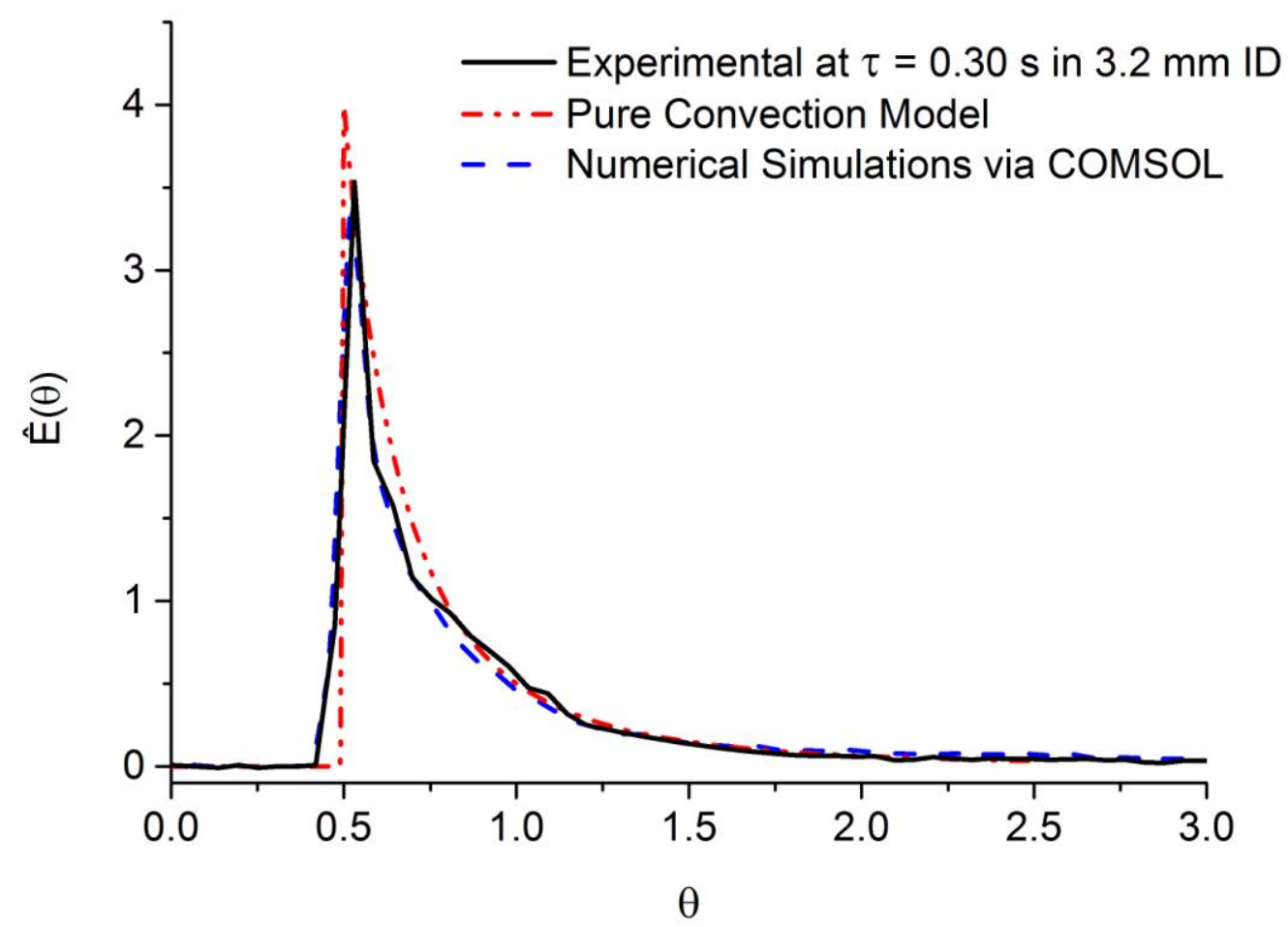

1

2 Figure 7: Residence Time Distribution in the presence of ultrasound-induced cavitation with negligible

3 Eckart acoustic streaming effects (sonication residence time of $0.30 \mathrm{~s}$ in the $3.2 \mathrm{~mm}$ I.D. capillary).

In the case presented in Figure 8, Eckart acoustic streaming effects are no longer negligible: vortices do form, even if they are underdeveloped compared to those observed in the same capillary at a sonication residence time of $3.0 \mathrm{~s}$. The experimental RTD differs significantly from that of the theoretical convection model, both in shape and width; however, owing to the limited extent of mixing, it does not take on the shape of the RTD presented in Figure 6 , which refers to a case in which vortices are more pronounced. In the case of underdeveloped vortices, the output of the model is extremely sensitive to input parameters (e.g. distance of the horn from the capillary wall), and this is probably the reason for the discrepancy observed between the experimental and the simulation results. Due to experimental error, not all parameters were kept exactly constant (e.g. the distance between the horn and capillary wall was measured within an error of $0.05 \mathrm{~mm}$ ). Small deviations between the model and experiments caused a more noticeable effect in this scenario compared to all others. The distance of the horn from the capillary wall was selected as a case study to investigate the sensitivity of the model to the input parameters and is discussed in the Supplementary Information. The discrepancy between simulation results and experimental data, 


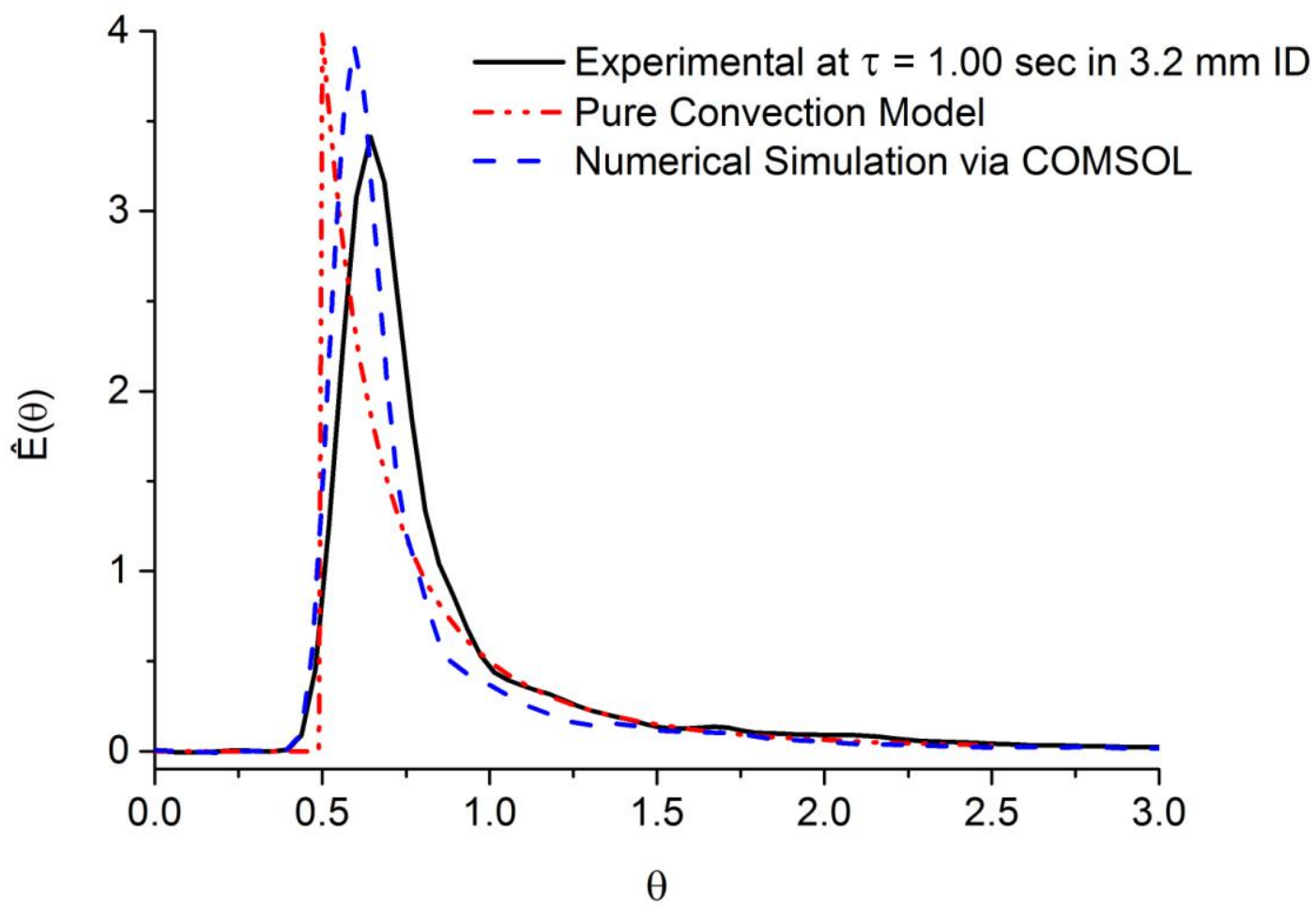

1

Figure 8: Residence Time Distribution in the presence of ultrasound-induced cavitation with mild Eckart acoustic streaming effects (sonication residence time of $1.00 \mathrm{~s}$ in the $3.2 \mathrm{~mm}$ I.D. capillary).

Generally, narrow RTDs are desired, since they lead to narrower crystal size distributions and more uniform growth due to a more homogeneous concentration profile. Enhanced mixing, which is promoted by acoustic vortices, should lead to more desirable CSDs. However, strong recirculation leads to backflow of the solution and some crystals may leave and re-enter the cavitation zone. Also, the residence time distribution of the crystals is different from that of the solution due to the lack of diffusive mass transport of crystals (Brownian motion is negligible). Some crystals travel rapidly through the system, along streamlines which bypass the vortices, while others remain inside them for prolonged periods of time. Hence, despite more homogeneous conditions (in terms of solute concentration) in the sonication region and narrower RTDs, the CSD does not necessarily improve by the introduction of acoustic streaming.

\subsection{Effect of Sonication Residence Time on Crystallization in 1.55 mm I.D. Capillary (negligible} acoustic streaming)

The mean residence time during which the solution is exposed to ultrasound was decreased by increasing the fluid flow rate $(Q=0.5,1.5,4.9 \mathrm{~mL} / \mathrm{min})$ in the $1.55 \mathrm{~mm}$ I.D. capillary tube; the corresponding values of $\tau_{S}$ were $3.0,1.0$ and $0.3 \mathrm{~s}$, respectively. The temperature of the solution was 
$110^{\circ} \mathrm{C}$, which corresponds to an initial supersaturation ratio of 2.12 . Figure 9 shows the yield against

2 the mean sonication residence time. Note that, as discussed previously, in the $1.55 \mathrm{~mm}$ I.D. capillary 3 tube acoustic stream is absent, so that the velocity field closely resembles that observed in silent laminar flow conditions.

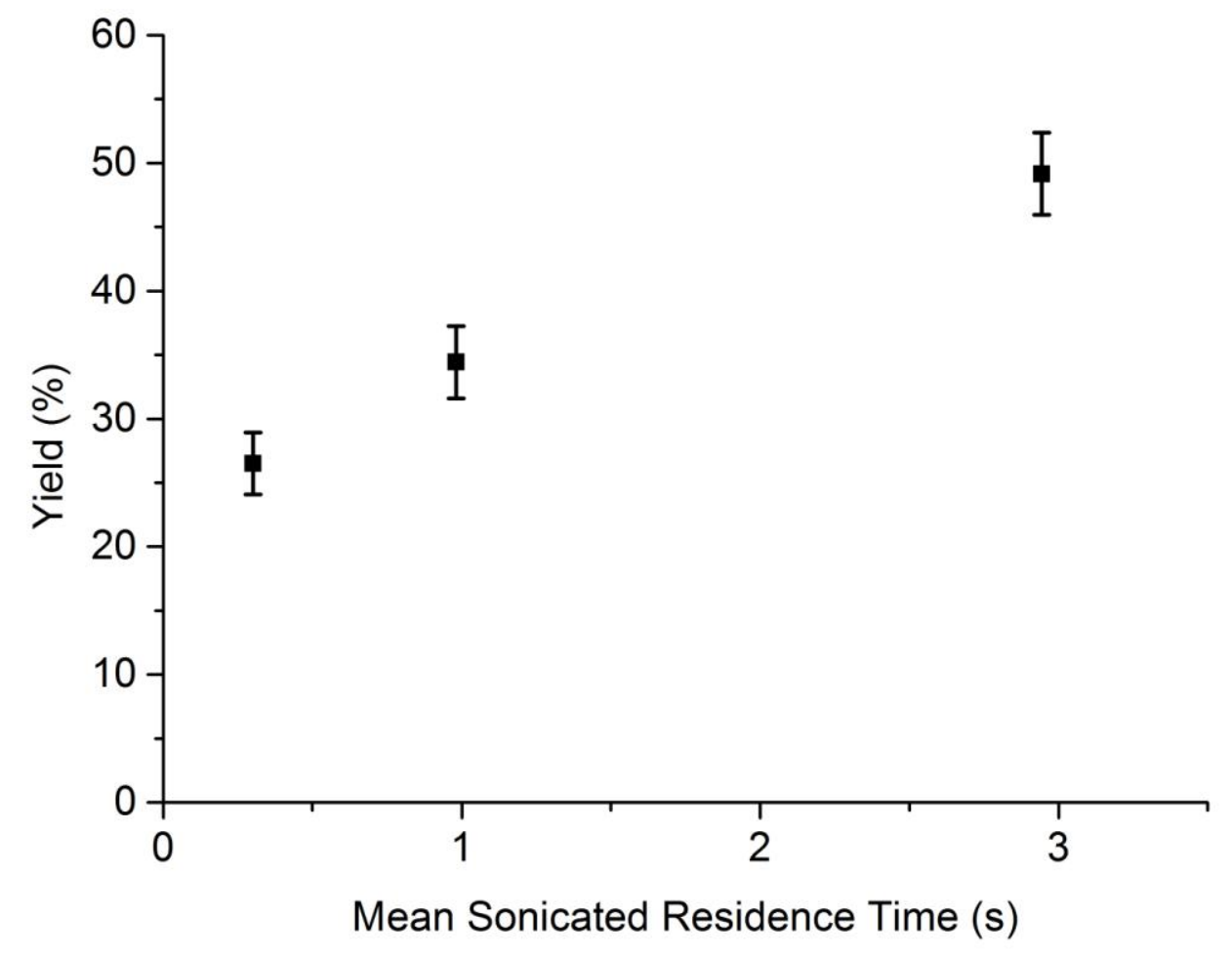

Figure 9: Effect of mean sonication residence time on crystal yield in the $1.55 \mathrm{~mm}$ I.D. capillary.

7 As reported in the literature $[43,46]$, the yield increases when the sonication residence time is 8 increased. The sonication volume inside the capillary was fixed and the cavitation activity within that 9 volume did not change when the fluid flow rate was varied. Nevertheless, as the sonication residence time $\tau_{S}$ increased, more crystalline material was generated owing to the longer time available for both ultrasound-enhanced nucleation and growth processes to act on the solution elements, as these flowed through the capillary. This explains the increase of crystal yield with $\tau_{S}$, the yield rising from $27 \%$ (for $\tau_{S}=0.3 \mathrm{~s}$ ) to $49 \%$ (for $\tau_{S}=3.0 \mathrm{~s}$ ).

One interesting observation is that the yield in the single-phase system investigated in this work is higher than that obtained in the droplet-based system considered in our previous work [42]. An explanation for this is that in this work the horn was located closer to the capillary wall, and so delivered more ultrasonic power to the adipic acid solution. Furthermore, bubble cavitation in milliand micro-channels is more vigorous at the sidewalls, where it is enhanced by the wall roughness 
1 (owing to bubble nuclei present in the crevices of the walls) [47]. The results also agree with the

2 findings of Arora et al. [48], who showed that cavitation occurred in acrylic polymer tubes with rough surfaces but did not in smooth ones. In the single-phase system that we are considering, there

4 is no hexane film shielding the capillary walls (in contrast with the two-phase droplet-based system 5 we previously studied [42]); hence, the presence of wall roughness leads to an increase in the number of bubbles available for cavitation.

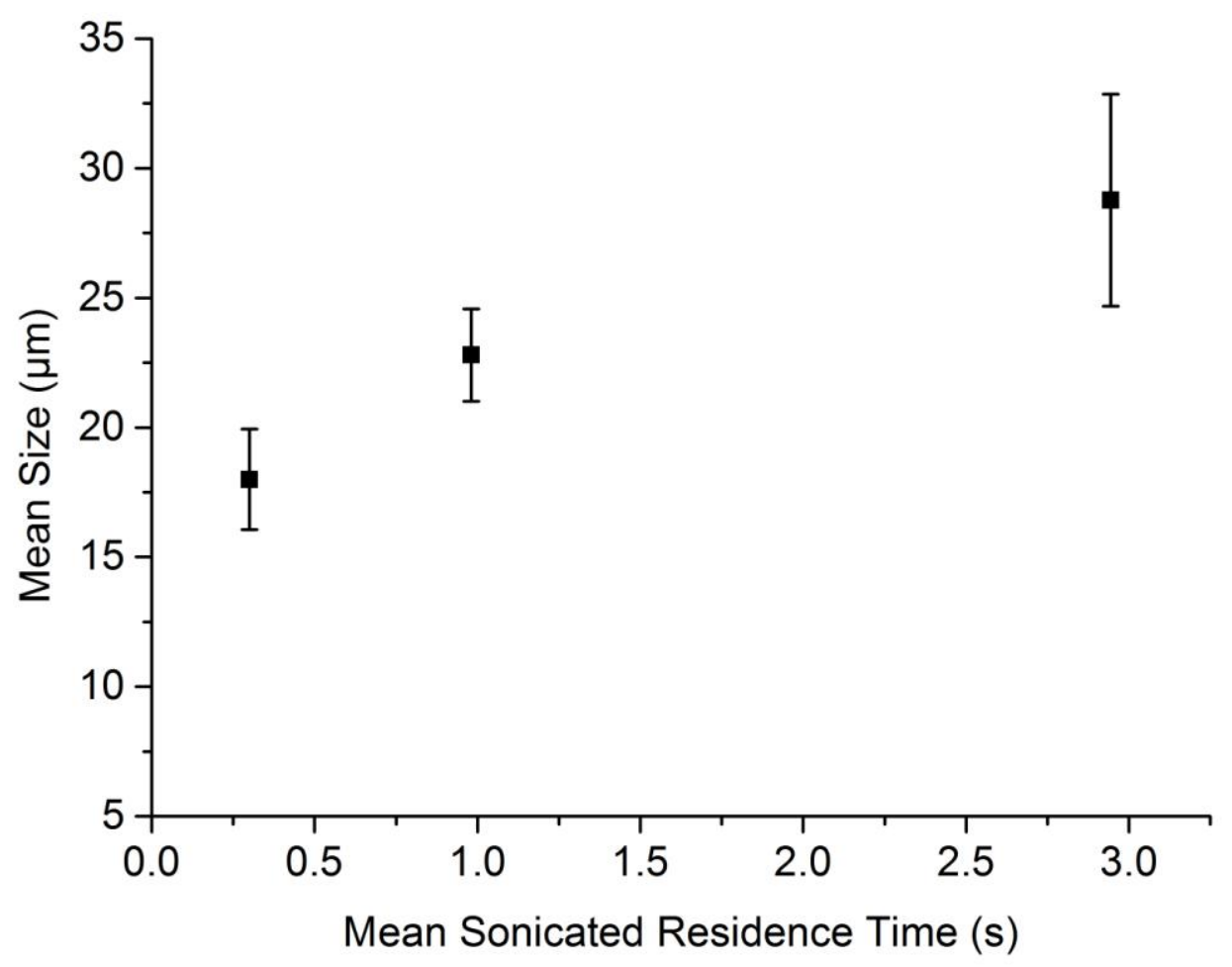

Figure 10: Effect of mean sonication residence time on mean crystal size in the $1.55 \mathrm{~mm}$ I.D. capillary. repeats (not the standard deviation of CSD which is given in supplementary information)

Figure 10 reports the mean size of the crystals $D_{4,3}$ obtained in the $1.55 \mathrm{~mm}$ I.D. capillary tube as a function of the mean sonication residence time. The microfluidic sonocrystallizer produces crystals with mean size ranging between $18 \mu \mathrm{m}$ and $29 \mu \mathrm{m}$ (which is consistent with our previous work [42]). A key finding in this system is that an increase in the mean sonication residence time leads to an increase in mean crystal size. The reason for this outcome is similar to that outlined for the increase in yield. As a crystal travels through the crystallizer, it grows; so, the longer this crystal spends in the crystallizer, the larger it becomes. This simple explanation, however, holds as long as changes in mean sonication residence time are not accompanied by changes in flow patterns, RTD 
1 and solute concentration profile. In the capillary we are currently discussing this is indeed the case,

2 but the same is not true in the $3.2 \mathrm{~mm}$ I.D. capillary tube, owing to acoustic streaming.

3

\subsection{Effect of Sonication Residence Time on Crystallization in $3.2 \mathrm{~mm}$ I.D. Capillary (significant} acoustic streaming)

In order to investigate the effect of acoustic streaming on crystallization, we increased the capillary size from $1.55 \mathrm{~mm}$ I.D. to $3.2 \mathrm{~mm}$ I.D.. As our numerical simulations suggest, in the case of $3.2 \mathrm{~mm}$ I.D. capillary, the effect of acoustic streaming is appreciable. To maintain the same sonication residence time as for the smaller capillary tube, we increased the flow rate to $Q=2,6.7,17.6 \mathrm{ml}$ / $\min$.

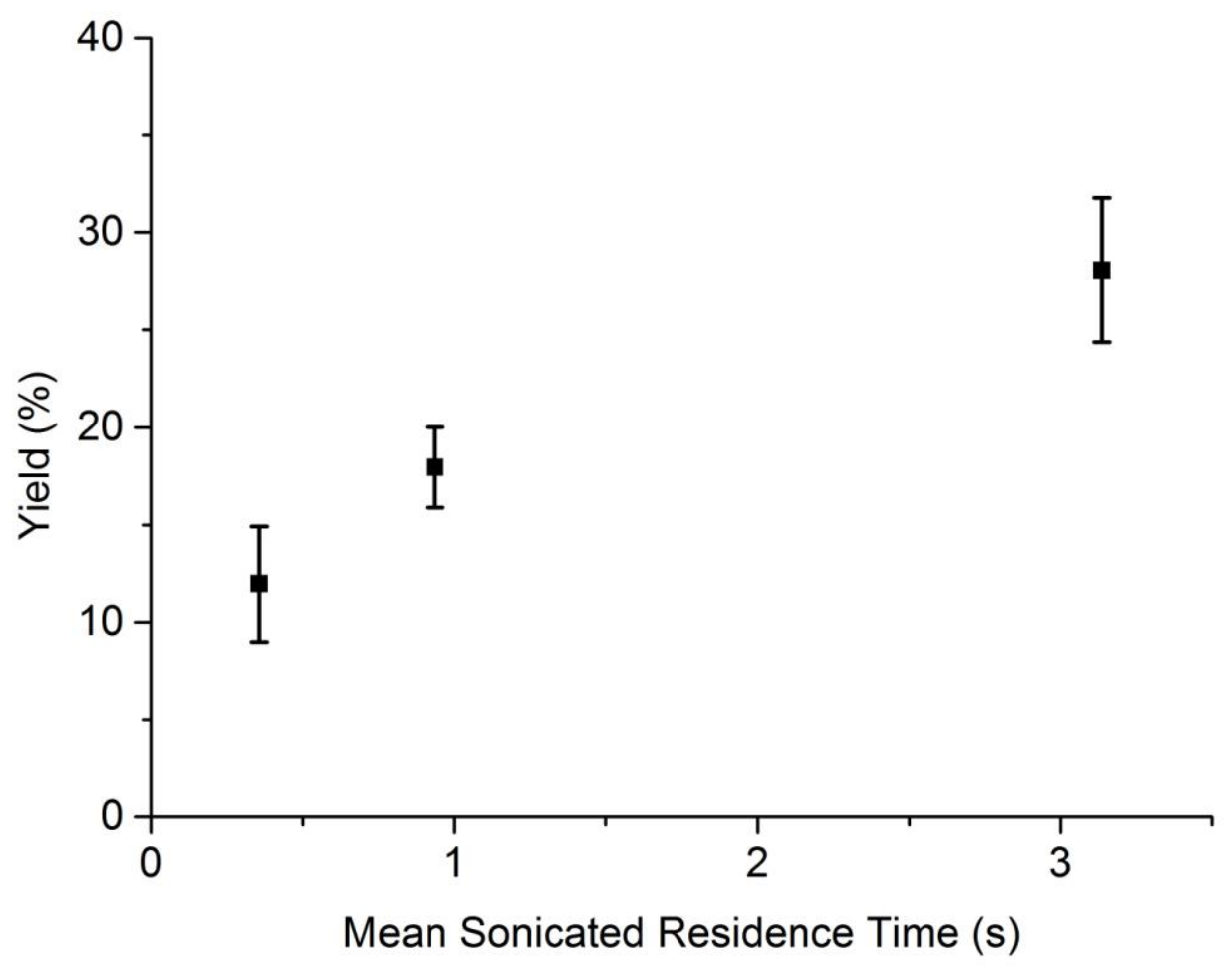

Figure 11: Effect of mean sonication residence time on crystal yield in the $3.2 \mathrm{~mm}$ ID capillary.

Figure 11 shows that also in the $3.2 \mathrm{~mm}$ I.D. capillary the yield increases with the mean sonication residence time. This trend can be explained with the same considerations presented for the capillary of smaller diameter. However, in the larger capillary, at any sonication residence time, the yield is smaller than that obtained in the smaller capillary at the same residence time. This may be due to the following three key differences between the two capillaries: 
- In the larger capillary, the ratio of surface area of the capillary to volume of fluid under the horn is smaller. This might seem insignificant, insofar as heterogeneous nucleation at the wall is negligible compared to the nucleation in the bulk of the solution. However, one must consider the availability of bubble nuclei for cavitation. Some of these come from the dissolved gas in the liquid bulk (in our case their concentration is reduced, since we used purified water for the solution), while others come from the gas pockets trapped in the crevices on the rough surface of the capillary walls. The higher the availability of bubble nuclei trapped at the walls, the more the cavitation activity. Therefore, one can expect higher cavitation activity (per unit volume of solution) in the smaller tube, and so higher yield.

- In the larger tube, acoustic streaming induces rapid mixing under the horn, which results in a back-mixing of the solution from downstream back to the sonicated volume. This results in a lower average solute concentration under the horn, which should result into a lower yield. In the smaller tube acoustic streaming is negligible and so the yield should be higher.

- By determining numerically the acoustic pressure field, we ensured that transient cavitation is present throughout the entire sonicated volume in both capillaries. To this end, we employed the transient cavitation threshold criterion outlined in [43]. In this paper, considering the same ultrasound source, we concluded that, at a frequency of $20 \mathrm{kHz}$, all bubbles undergo transient cavitation when the amplitude of the acoustic pressure is higher than about 2 bar. In the larger capillary, the acoustic pressure does not drop below 2 bar, and so transient cavitation occurs everywhere within the sonicated volume (furthermore, the bubble collapse frequency in the solution bulk is expected to be the same for both capillaries). So, from this point of view, the behaviour of the two capillaries is quite similar.

Nevertheless, the transient cavitation threshold criterion does not consider the intensity of the bubble collapse, which is expected to decrease as the distance from the horn increases (and to affect the local rate of nucleation). In the larger capillary, there is a region with higher acoustic pressure amplitude, a region with similar acoustic pressure amplitude, and a region with lower acoustic pressure amplitude than that present in the smaller capillary. The first of these is the region of the capillary closest to the horn, whilst the last of these is the region of the capillary furthest from the horn. Therefore, in the larger capillary there should be three regions: one in which the bubble collapse intensity is higher than that present in the smaller capillary, one in which it is similar, and one in which it is lower. From this point of view, the two capillaries differ. This difference cannot be eliminated, but by keeping the distance between the centreline of the capillaries and the tip of the horn constant, we tried to minimise its effect. 
To investigate the effect of this difference (in particular, of the regions which are closest and furthest from the horn) on crystallization, one would require a (reliable) model. Currently, the effect of bubble collapse intensity on crystallization, and in particular on nucleation, is unclear; in fact, reliable models for sonocrystallization are absent. So, this analysis, for the time being, cannot be conducted.

The effect of $\tau_{s}$ on mean crystal size is different when acoustic streaming is present, as can be seen in Figure 12. In this case, in contrast with the $1.55 \mathrm{~mm}$ I.D. capillary, the mean size decreases with an increase in mean sonication residence time. We have found a statically significant difference between each mean crystal size, which shows the existence of a trend despite the large error bars ( $p<2.1 \%$; further details are given in the Supplementary Information). At high values of $\tau_{s}-$ in the presence of strong acoustic streaming vortices - the value of the mean crystal size is smaller than that at low values of $\tau_{s}$ - in the absence of vortices. This is possible because changes in the mean sonication residence time are, in this case, accompanied by changes in flow patterns, RTD and solute concentration profile. At large $\tau_{s}$, the significant increase in mixing leads to a lower solute concentration in the sonication region of the capillary due to back-mixing of the solution, which is expected to result into a lower growth rate. This effect, which tends to produce smaller crystals, is however counteracted by the longer time that crystals can grow in the sonication region of the capillary, which tends to increase the crystal size. So, predicting the functional dependence of mean crystal size on mean sonication residence time is not trivial. To analytically answer the question of whether crystal size would increase or decrease with mean sonication residence time, one should know, in addition to the flow pattern, the kinetics of crystal nucleation and growth in the presence of ultrasound; these constitutive relations are still unavailable. The experimental results, on the other hand, reveal that, for this particular case, the decrease in growth rate is the dominant effect. However, we must note that such conclusion has no general validity, being system dependent. 


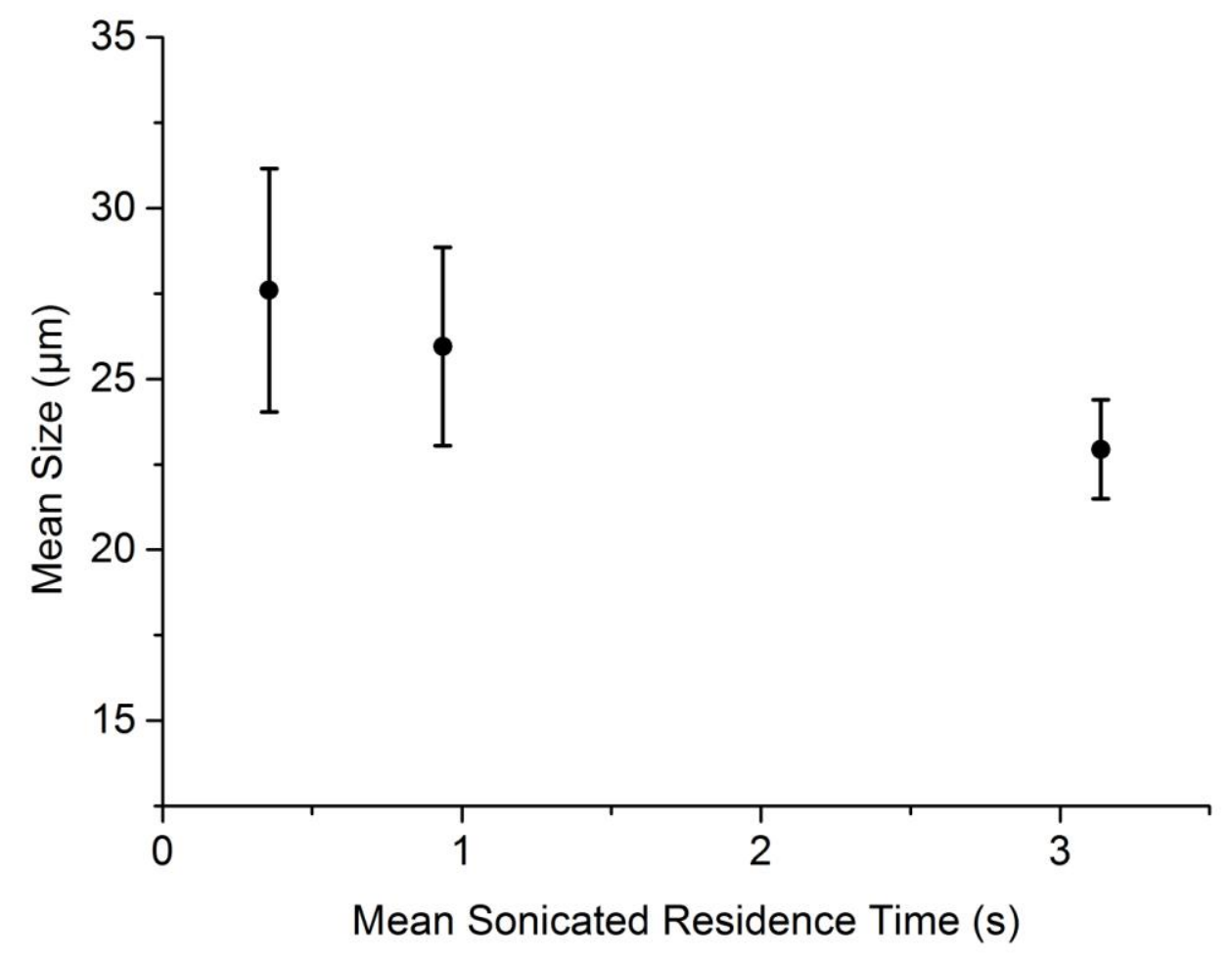

Figure 12: Effect of mean sonication residence time on mean crystal size in the $3.2 \mathrm{~mm} I D$ capillary. The error bars represent one standard deviation in mean crystal size across different experimental repeats (not the standard deviation of CSD which is given in supplementary information)

3 The important insight that these experimental results provide is that the performance of the 4 crystallizer can be strongly affected by how ultrasound is applied and how significant acoustic 5 streaming is. The action of acoustic streaming, in turn, strongly depends on the operating conditions 6 and the system geometry: a relatively small increase of the size of the capillary tube can have 7 significant consequences on the system performance. To fully exploit the potential that ultrasound 8 offers in terms of process control and performance enhancement, one needs to design and operate 9 the sonocrystallizer appropriately. Doing so requires a systematic approach which should be guided by modelling, as we have done in this work.

\section{Conclusions}

12 This study concerns the characterization of mixing and crystallization under the effect of cavitation 13 and acoustic streaming induced by ultrasound. Experiments and numerical simulations were carried out for different operating conditions with two capillary tubes to determine residence time 
distributions, flow patterns, and influence of acoustic streaming on the velocity field and crystallization performance.

The design of the crystallizer was based on the insight offered by numerical modelling of the system. Having determined numerically the spatial profile of the wave pressure amplitude, we were then able to identify the regions of the system within which inertial cavitation occurred. This information was then used to design the crystallizer (in terms of size of the capillary tube and distance from the source of ultrasound). Such methodology allowed us to ensure that the proposed design would achieve the desired level of cavitation, which is the driving force behind the enhancement of crystallization rates (in particular, of the nucleation process).

We also considered the effect that ultrasound has on the flow field. We showed that increasing the size of the capillary can have multiple and interacting effects on the system performance and crystal properties. Changing either the capillary size or the solution flow rate can lead to radical changes in acoustic streaming, which in turn can affect significantly the solute concentration profile inside the capillary, as was shown by the RTD study. We considered two capillaries with diameters of the same order of magnitude $(1.55 \mathrm{~mm}$ and $3.2 \mathrm{~mm}$ ) operated in similar conditions, observing that their performance was quite different. In the smaller capillary, acoustic streaming did not influence flow patterns and RTD, and raising the mean sonication residence time resulted into an increase in mean crystal size. In the (slightly) larger capillary the opposite was observed: acoustic streaming influenced flow patterns and RTD when the solution flow rate was sufficiently low and increasing the mean sonication residence time resulted into a decrease in mean crystal size. Modelling of the fluid dynamics and ultrasound (as done in this work), if not of the crystallization process (which, for the time being, still appears to be not viable), can aid considerably in overcoming these challenges.

\section{Bibliography}

[1] J. Chen, B. Sarma, J.M.B.M.B. Evans, A.S.S. Myerson, Pharmaceutical crystallization, Cryst. Growth Des. 11 (2011) 887-895.

[2] G. Ruecroft, D. Hipkiss, T. Ly, N. Maxted, P.W. Cains, Sonocrystallization: the use of ultrasound for improved industrial crystallization, Org. Process Res. Dev. 9 (2005) 923-932.

[3] M. Sultana, K.F.F. Jensen, Microfluidic continuous seeded crystallization: Extraction of growth kinetics and impact of impurity on morphology, Cryst. Growth Des. 12 (2012) 6260-6266.

[4] M.O. Besenhard, P. Neugebauer, C.-D. Ho, J.G. Khinast, Crystal Size Control in a Continuous 
Tubular Crystallizer, Cryst. Growth Des. 15 (2015) 1683-1691.

[5] R.L. Hartman, K.F. Jensen, Microchemical systems for continuous-flow synthesis, Lab a Chip Miniaturisation Chem. Biol. 9 (2009) 2495-2507.

[6] K. Wu, S. Kuhn, Strategies for solids handling in microreactors, Chim. Oggi/Chemistry Today. 32 (2014) 62-66.

[7] D. Fernandez Rivas, S. Kuhn, Synergy of Microfluidics and Ultrasound, Top. Curr. Chem. 374 (2016) 70.

[8] W. Benzinger, U. Schygulla, M. Jäger, K. Schubert, Anti fouling investigations with ultrasound in a microstructured heat exchanger, in: ECI Symp. Ser. Proc. 6th Inter. Conf. Heat Exch. Fouling Clean., 2010.

[9] R.J. Wood, J. Lee, M.J. Bussemaker, A parametric review of sonochemistry: Control and augmentation of sonochemical activity in aqueous solutions, Ultrason. Sonochem. 38 (2017) 351-370.

[10] T.J. Mason, Ultrasound in synthetic organic chemistry, Chem. Soc. Rev. 26 (1997) 443-451.

[11] N.S. Deora, N.N. Misra, a Deswal, H.N. Mishra, P.J. Cullen, B.K. Tiwari, Ultrasound for Improved Crystallisation in Food Processing, Food Eng. Rev. 5 (2013) 36-44.

[12] L.D.L.S. Castillo-Peinado, M.D. Luque de Castro, The role of ultrasound in pharmaceutical production: sonocrystallization, J. Pharm. Pharmacol. (2016) 1249-1267.

[13] D. Rossi, A. Gavriilidis, S. Kuhn, M.A. Candel, A.G. Jones, C. Price, L. Mazzei, Adipic Acid Primary Nucleation Kinetics from Probability Distributions in Droplet-Based Systems under Stagnant and Flow Conditions, Cryst. Growth Des. (2015).

[14] M.D.D. Luque de Castro, F. Priego-Capote, M.D. de Castro, F. Priego-Capote, M.D.D. Luque de Castro, F. Priego-Capote, Ultrasound-assisted crystallization (sonocrystallization), Ultrason. Sonochem. 14 (2007) 717-724.

[15] L.J. McCausland, Sonocrystallization - the use of power ultrasound to control and manipulate the nucleation of crystals, Chem Ing Tech. 73 (2001) 717-719.

[16] O. Narducci, A.G.G. Jones, E. Kougoulos, G.J. a, E. Kougoulos, A.G.G. Jones, E. Kougoulos, Continuous crystallization of adipic acid with ultrasound, Chem. Eng. Sci. 66 (2011) 10691076. 
[17] M. Furuta, K. Mukai, D. Cork, K. Mae, Continuous crystallization using a sonicated tubular system for controlling particle size in an API manufacturing process, Chem. Eng. Process. Process Intensif. 102 (2016) 210-218.

[18] M. Jiang, C.D.C.D. Papageorgiou, J. Waetzig, A. Hardy, M. Langston, R.D.R.D. Braatz, Indirect Ultrasonication in Continuous Slug-Flow Crystallization, Cryst. Growth Des. 15 (2015) 24862492.

[19] R.J.P. Eder, S. Schrank, M.O. Besenhard, E. Roblegg, H. Gruber-Woelfler, J.G. Khinast, Continuous Sonocrystallization of Acetylsalicylic Acid (ASA): Control of Crystal Size, Cryst. Growth Des. 12 (2012) 4733-4738.

[20] H. Siddique, C.J. Brown, I. Houson, A.J. Florence, Establishment of a Continuous Sonocrystallization Process for Lactose in an Oscillatory Baffled Crystallizer, Org. Process Res. Dev. 19 (2015) 1871-1881.

[21] B. Gielen, T. Claes, J. Janssens, J. Jordens, L.C.J. Thomassen, T. Van Gerven, L. Braeken, Particle Size Control during Ultrasonic Cooling Crystallization of Paracetamol, Chem. Eng. Technol. 40 (2017) 1300-1308.

[22] J. Jordens, E. Canini, B. Gielen, T. Van Gerven, L. Braeken, Ultrasound Assisted Particle Size Control by Continuous Seed Generation and Batch Growth, 2017.

[23] O. Louisnard, J. González-garca, Acoustic Cavitation, in: H. Feng, G. Barbosa-Canovas, J. Weiss (Eds.), Ultrasound Technol. Food Bioprocess., 2011.

[24] L.J. Briggs, Limiting Negative Pressure of Water, J. Appl. Phys. 21 (1950) 721-722.

[25] M. Dular, O. Coutier-Delgosha, Thermodynamic effects during growth and collapse of a single cavitation bubble, J. Fluid Mech. 736 (2013) 44-66.

[26] K.S. Suslick, Sonochemistry, Science (80-. ). 247 (1990) 1439-1445.

[27] Y.T. Didenko, W.B. McNamara lii, K.S. Suslick, Hot spot conditions during cavitation in water, J. Am. Chem. Soc. 121 (1999) 5817-5818.

[28] S.J. Lighthill, Acoustic streaming, J. Sound Vib. 61 (1978) 391-418.

[29] N. Riley, STEADY STREAMING, Annu. Rev. Fluid Mech. 33 (2001) 43-65.

[30] S. Boluriaan, P.J. Morris, Acoustic Streaming: From Rayleigh to Today, Int. J. Aeroacoustics. 2 
(2003) 255-292.

[31] M. Wiklund, R. Green, M. Ohlin, Acoustofluidics 14: Applications of acoustic streaming in microfluidic devices, Lab Chip. 12 (2012) 2438-2451.

[32] H.-D. Xi, H. Zheng, W. Guo, A.M. Ganan-Calvo, Y. Ai, C.-W. Tsao, J. Zhou, W. Li, Y. Huang, N.-T. Nguyen, S.H. Tan, Active droplet sorting in microfluidics: a review, Lab Chip. 17 (2017) 751771.

[33] J.J. O'Sullivan, M. Park, J. Beevers, R.W. Greenwood, I.T. Norton, Applications of ultrasound for the functional modification of proteins and nanoemulsion formation: A review, Food Hydrocoll. 71 (2017) 299-310.

[34] J.W. Mullin, Crystallization, 2001.

[35] F.B. Jensen, W.A. Kuperman, M.B. Porter, H. Schmidt, J.F. Bartram, Computational Ocean Acoustics, J. Acoust. Soc. Am. 97 (1995) 3213.

[36] R. Jamshidi, B. Pohl, U. a U.A. Peuker, G. Brenner, Numerical investigation of sonochemical reactors considering the effect of inhomogeneous bubble clouds on ultrasonic wave propagation, Chem. Eng. J. 189-190 (2012) 364-375.

[37] K.W. Commander, A. Prosperetti, Linear Pressure Waves In Bubbly Liquids: Comparison Between Theory And Experiments, J. Acoust. Soc. Am. 85 (1989).

[38] S. Labouret, J. Frohly, Bubble size distribution estimation via void rate dissipation in gas saturated liquid. Application to ultrasonic cavitation bubble fields, 2002.

[39] K. Matsuda, T. Kamakura, M. Maezawa, Local control of eckart streaming near focus of concave ultrasound source with two coaxially arranged transducers, Japanese J. Appl. Physics, Part 1 Regul. Pap. Short Notes Rev. Pap. 45 (2006) 4448-4452.

[40] J. Grau-Bové, M. Strlič, L. Mazzei, Applicability of a drift-flux model of aerosol deposition in a test tunnel and an indoor heritage environment, Build. Environ. 106 (2016) 78-90.

[41] O. Levenspiel, Chemical reaction engineering, 3rd ed., John Wiley \& Sons, New York, 1999.

[42] D. Rossi, R. Jamshidi, N. Saffari, S. Kuhn, A. Gavriilidis, L. Mazzei, Continuous-Flow Sonocrystallization in Droplet-Based Microfluidics, Cryst. Growth Des. 15 (2015) 5519-5529.

[43] R. Jamshidi, D. Rossi, N. Saffari, A. Gavriilidis, L. Mazzei, Investigation of the Effect of 
Ultrasound Parameters on Continuous Sonocrystallization in a Millifluidic Device, Cryst. Growth Des. 16 (2016) 4607-4619.

[44] P. J W Roberts, D. R Webster, Turbulent diffusion, 2003.

[45] M. Liu, Computational study of convective-diffusive mixing in a microchannel mixer, Chem. Eng. Sci. v. 66 (2011).

[46] T. T. H. Nguyen, A. Khan, L. Mir Bruce, C. Forbes, R. O'Leary, C. J. Price, The Effect of Ultrasound on the Crystallisation of Paracetamol in the Presence of Structurally Similar Impurities, 2017.

[47] A. Ozcelik, D. Ahmed, Y. Xie, N. Nama, Z. Qu, A.A. Nawaz, T.J. Huang, An acoustofluidic micromixer via bubble inception and cavitation from microchannel sidewalls, Anal. Chem. 86 (2014) 5083-5088.

[48] M. Arora, C.D. Ohl, K.A. Mørch, Cavitation inception on microparticles: A self-propelled particle accelerator, Phys. Rev. Lett. 92 (2004) 174501. 


\title{
Supplementary Information
}

\section{Effect of Acoustic Streaming on \\ Continuous Flow Sonocrystallization in Millifluidic Channels}

\author{
Gleb Valitov, Rashid Jamshidi, Damiano Rossi, Asterios Gavriilidis, Luca Mazzei \\ University College London, Department of Chemical Engineering, Torrington Place, London WC1E TJE, UK \\ *Corresponding author: I.mazzei@ucl.ac.uk
}

\section{Table of Contents}

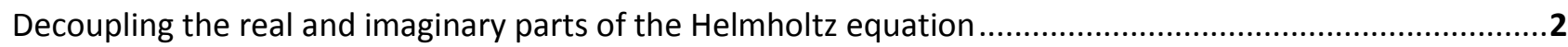

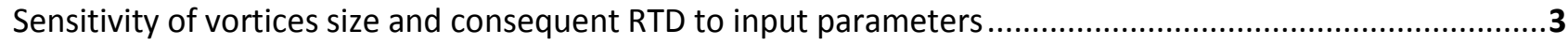

Information about crystal size distribution and crystal shape ................................................................

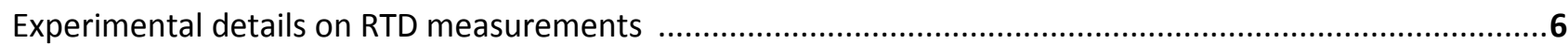

Magnitude of the velocity inside each capillary and sonication residence time ...........................................

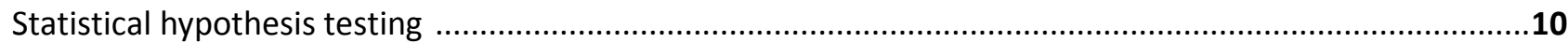




\section{Decoupling the real and imaginary parts of the Helmholtz equation}

Eq. (3.2) of the main article should be solved using the complex wave number defined in Eq. (3.3). The latter provides a field for the acoustic pressure amplitude which is complex [1],[2]. If we assume:

$P=\mathfrak{R}(P)+i \mathfrak{I}(P)=P_{r}+i P_{i}$

and

$k_{m}^{2}=\mathfrak{R}\left(k_{m}^{2}\right)+i \mathfrak{I}\left(k_{m}^{2}\right)=K_{r}+i K_{i}$

and since the Laplacian is a linear operator, we can decompose the Helmholtz equation (eq. (3.2)) into the following two coupled differential equations [3]:

$\nabla^{2} P_{r}+K_{r} P_{r}-K_{i} P_{i}=0$

and

$\nabla^{2} P_{i}+K_{r} P_{i}+K_{i} P_{r}=0$

in which

$K_{r}=\left(\frac{\omega}{c}\right)^{2}\left(1+\frac{4 \pi c^{2} n_{b} R_{0}\left(\omega_{0}^{2}-\omega^{2}\right)}{\left(\omega_{0}^{2}-\omega^{2}\right)^{2}+(2 \beta \omega)^{2}}\right)$

and

$K_{i}=\left(\frac{\omega}{c}\right)^{2}\left(-\frac{4 \pi c^{2} n_{b} R_{0}(2 \beta \omega)}{\left(\omega_{0}^{2}-\omega^{2}\right)^{2}+(2 \beta \omega)^{2}}\right)$

After computing the two fields, one uses the magnitude of the real part of the pressure amplitude, $P_{r}$, to calculate the source term of the acoustic streaming in Eq. 3.8. 


\section{Sensitivity of acoustic streaming to horn position}

In Section 4.3, we discuss the effect that acoustic streaming has on RTDs. A good match between numerical modelling and experiments is observed for all cases. Nevertheless, the agreement between the two is the weakest in the transitional regime where the onset of acoustic streaming occurs (at a sonication residence time of $1.0 \mathrm{~s}$ in the $3.2 \mathrm{~mm}$ I.D. capillary). This specific sonication residence time is the most sensitive to the experimental conditions employed, in particular to the distance between the horn and the capillary wall or between the power output and the horn. Let us consider the former as a model case.

In the model, we vary the distance between the horn and the capillary wall from 0.5 to $0.55 \mathrm{~mm}$ (the difference between the two is the experimental error). The resulting streamlines are displayed in Figure S1. It can be observed that for cases (a) to (d) and (f) there is no difference in the model predictions. In these cases, the slight change in the distance between the horn and capillary (indirect way to change ultrasonic power) has no significant effect, because this power is either sufficiently high to promote fully developed vortices or low enough to have no significant effect. However, in case (e) the size of the vortices is larger when the horn is closer to the capillary. This is expected: the closer the capillary is to the horn, the higher the acoustic power is and the stronger the acoustic radiation force is. Since the vortices are only starting to develop, their size is strongly dependent on the acoustic conditions.

Owing to the high sensitivity of the vortices size to the operational conditions in the case of onset of acoustic streaming (case (e) in Figure S1), the experimental error and the modelling assumptions made have a far more significant effect on the resulting RTD. Figure S2 shows how the RTD changes when the distance between the horn and the capillary wall is varied. Three cases were considered, all referring to conditions in which acoustic streaming is at the onset. We can see that slight variations (within experimental error) of the horn-wall distance lead to noticeable changes in the RTD. Other parameters which are either estimated in the model (e.g., mean bubble size) or measured experimentally (e.g., calorimetric power of the horn) all can have an effect of the RTD obtained numerically. This effect is negligible in the cases with fully developed vortices or no vortices at all. However, in the case of onset of streaming, the errors in the input parameters lead to a worse match between the experimental results and the model predictions.

(a)

(d)

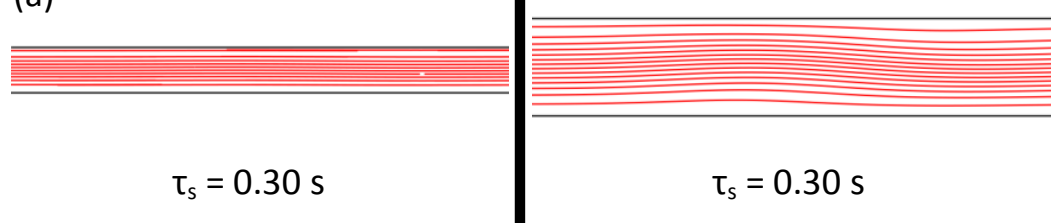

(b)

(e)

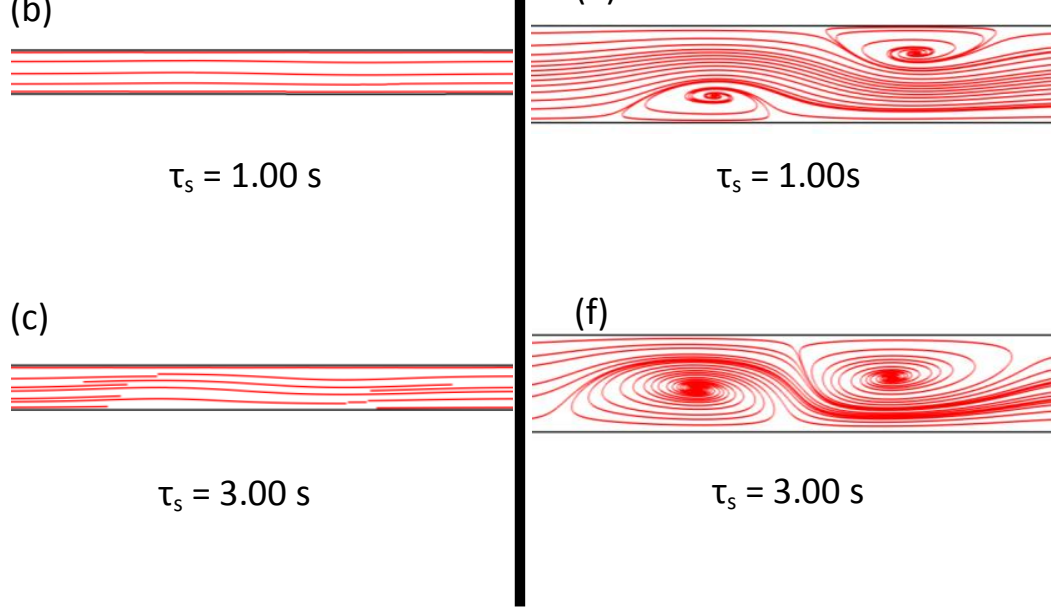


(a)

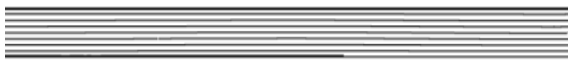

$$
\tau_{\mathrm{s}}=0.30 \mathrm{~s}
$$

(b)

\begin{tabular}{|c|c|}
\hline & $\tau_{\mathrm{s}}=1.00 \mathrm{~s}$ \\
\hline (c) & \\
\hline
\end{tabular}

$\tau_{\mathrm{s}}=3.00 \mathrm{~s}$ (d)

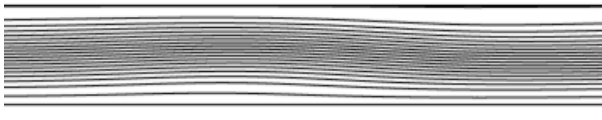

$$
\tau_{\mathrm{s}}=0.30 \mathrm{~s}
$$

(e)

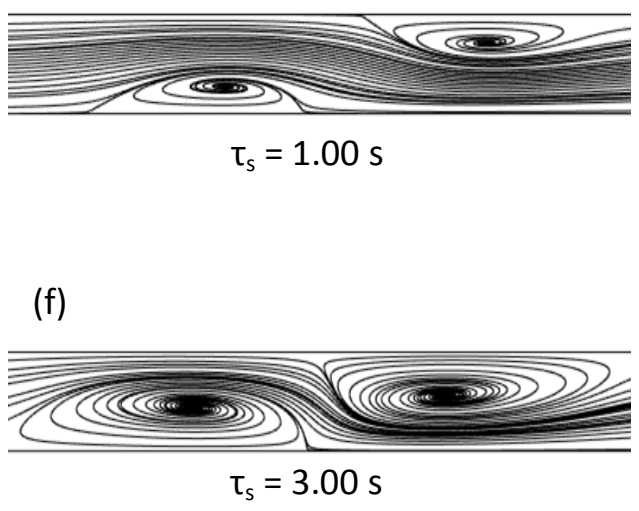

Figure S1: Streamlines inside the capillaries at different mean sonicated residence times at two different distances between the horn and the outer capillary wall: I) $d_{\text {horn }}=0.55 \mathrm{~mm}$ and II) $d_{\text {horn }}=0.5 \mathrm{~mm}$. In the $1.5 \mathrm{~mm}$ I.D. capillary the flow rates are (a) $4.8 \mathrm{ml} / \mathrm{min}$, (b) $1.5 \mathrm{ml} / \mathrm{min}$ and (c) $0.5 \mathrm{ml} / \mathrm{min}$; in the $3.2 \mathrm{~mm}$ I.D. capillary they are (d) $17.6 \mathrm{ml} / \mathrm{min}$, (e) $6.7 \mathrm{ml} / \mathrm{min}$ and (f) $2 \mathrm{ml} / \mathrm{min}$.

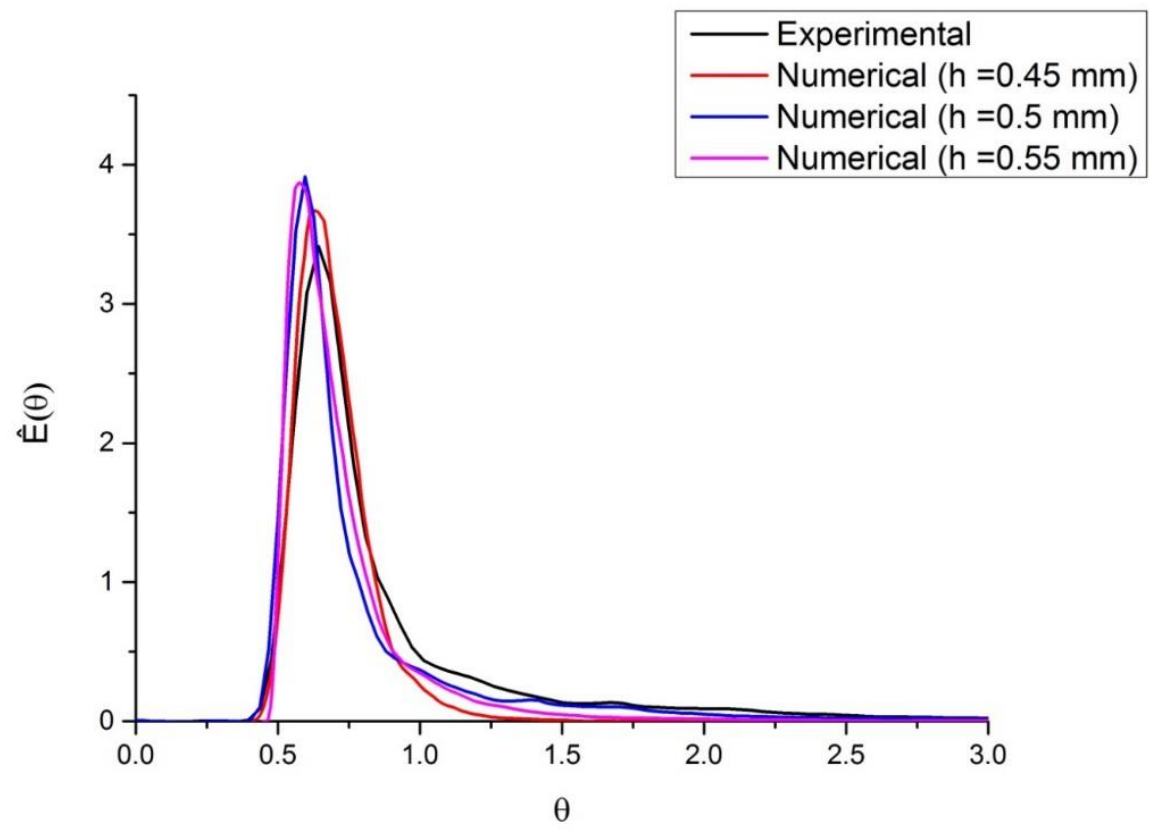

Figure S2: Residence time distribution for different distances between the horn and the capillary wall in the transitional regime where onset of acoustic streaming vortices occurs. The selected range for $d_{\text {horn }}$ (from 0.45 to $0.55 \mathrm{~mm}$ ) is representative of our experimental error in measuring this distance. 


\section{Information about crystal size distribution and crystal shape}

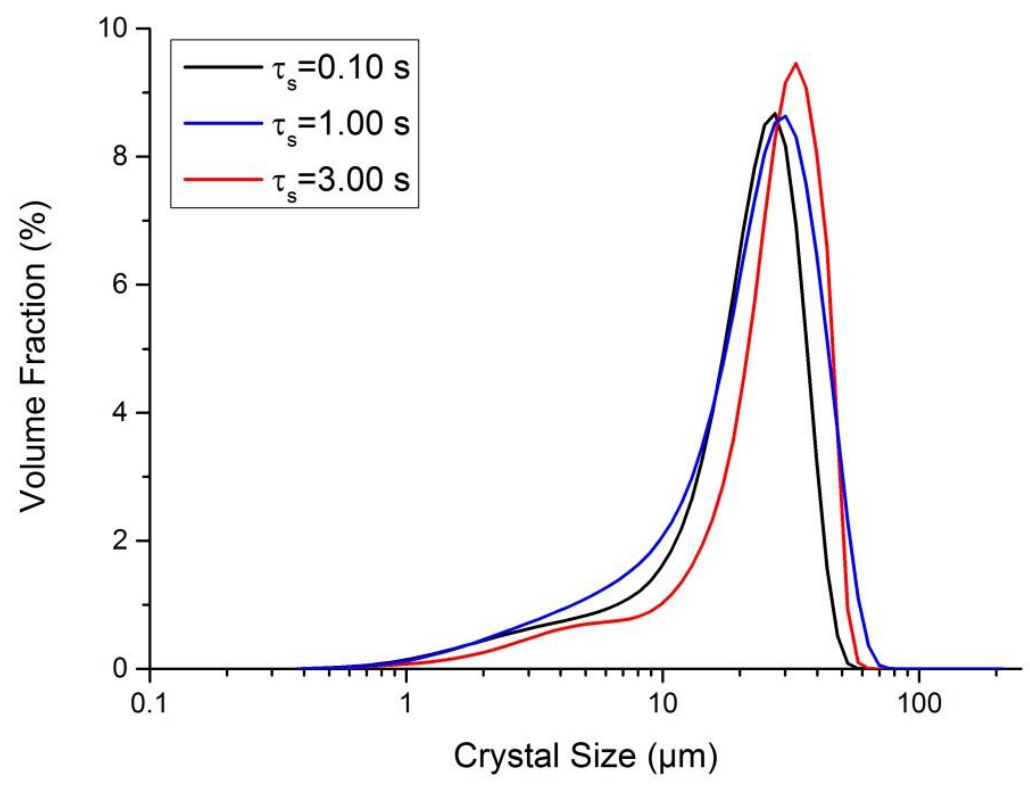

Figure S3: Crystal size distribution for the $1.55 \mathrm{~mm}$ I.D. capillary tube at the mean sonication residence time, $\tau_{s}$, indicated. Each distribution is an average of three measurements of one experimental repeat.

Figure S3 shows sample crystal size distributions (CSDs) for three different experiments in the $1.55 \mathrm{~mm}$ I.D. capillary. Such distributions are characteristic of all the CSDs obtained experimentally. Only one peak is observed. In the cases where the CSD was bimodal, the results were disregarded, since the bimodal shape is an indication of crystal growth occurring on the filter paper or of failed de-aggregation of crystals prior to size measurements. We did not find a correlation between the standard deviation of the distribution and the influence of ultrasound (both in terms of sonication residence time and acoustic streaming).

The shape of the crystals was observed via a microscope with $\times 20$ magnification lens. Different shapes were observed. At silent conditions (figure S4A), the crystals have uniform hexagonal shape, which is also reported in the literature [4]. On the other hand, under the effect of ultrasound (figure S4B) the shape of crystals becomes irregular and there is no evidence which suggests that the crystals have a particular shape (differently from what was observed in the absence of ultrasound).

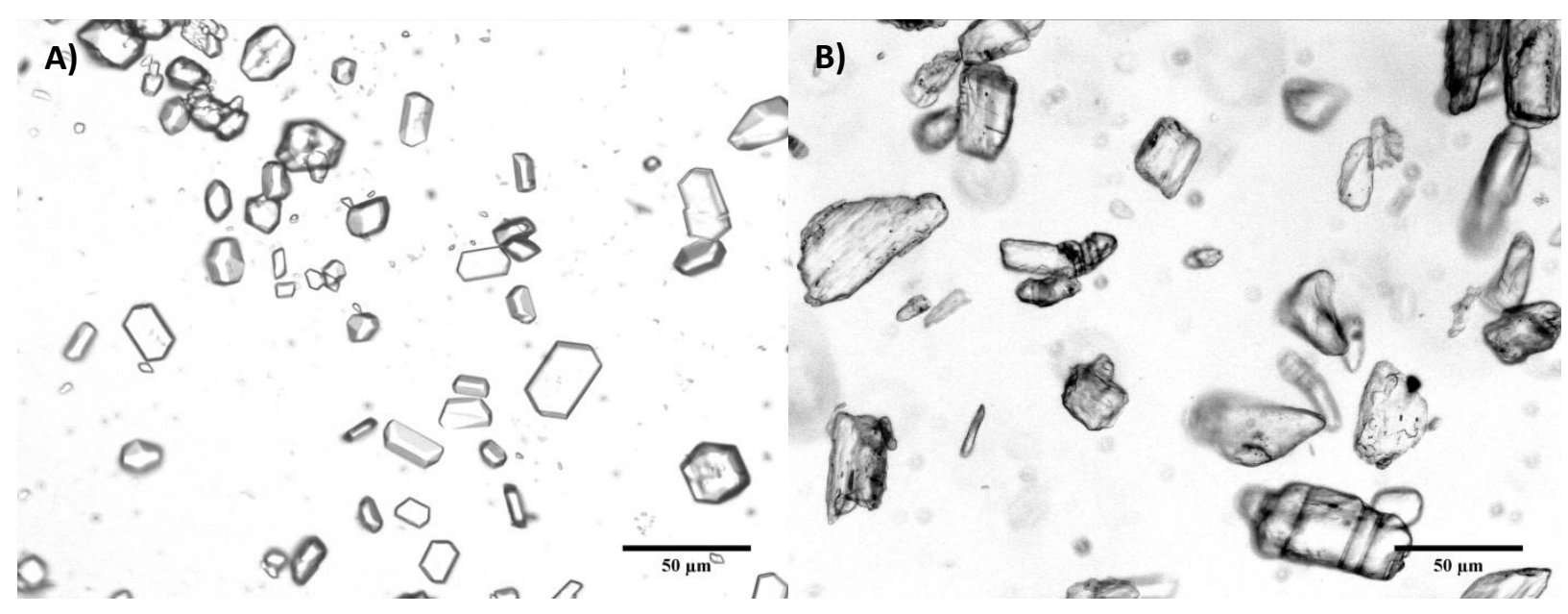

Figure S4: Crystal shape in the absence (A) and presence (B) of ultrasound. 


\section{Experimental details on RTD measurements}

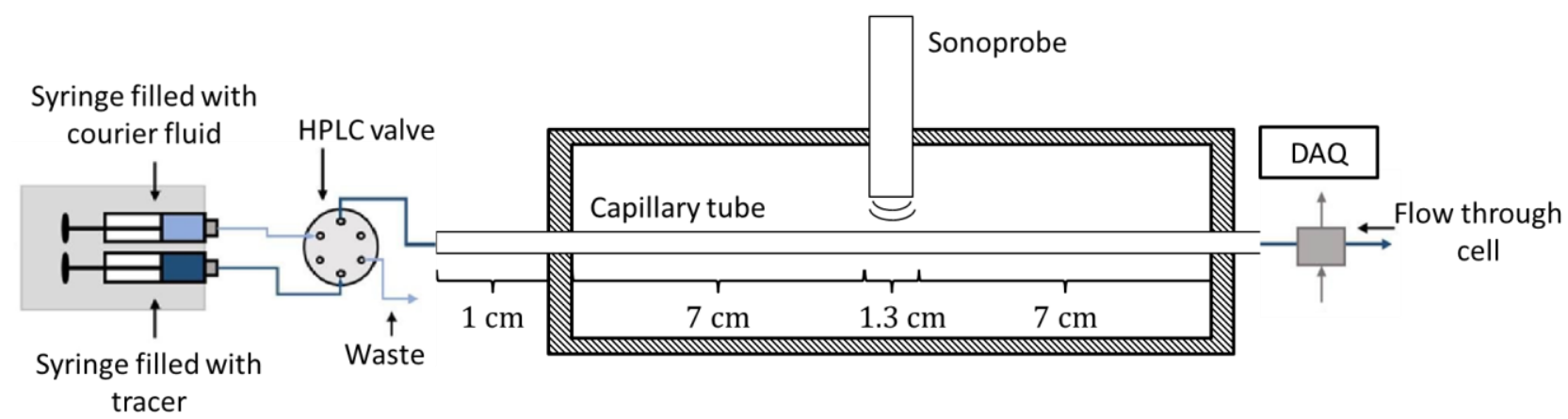

Figure S5: Schematic of the RTD experimental set-up. (Abbreviations: DAQ - Data Acquisition Board; HPLC valve - High Performance Liquid Chromatography Valve, used for step change in concentration of tracer mater).

The methodology outlined in the article was used to measure the residence time distribution (RTD) of the nucleation section, which included the additional $15 \mathrm{~cm}$ of capillary that is not located under the horn tip. These additional $15 \mathrm{~cm}$ were also included in the numerical simulations for comparative purposes. Alternatively, one could model only the sonicated region and, for comparison, apply deconvolution to calculate the RTD of the sonicated section alone using the experimental data. The latter methodology was not applied here, because in the presence of acoustic streaming the affected region is larger than the 1.3 $\mathrm{cm}$ domain under the horn.

The flow-through measurement cell consisted of two 3D printed parts kept together with bolts, as displayed in Figure S6. The surface of the flow cell which faces the capillary and fibre optic cables was covered with a thin layer of aluminium foil to prevent light from escaping through the flow cell. We noticed that adding the aluminium foil reduced significantly the background noise during the measurements. For each (different) capillary, we manufactured a separate flow cell. Such design allowed us to measure the tracer concentration directly after the sonicated section. We verified that the Beer-Lambert law applies for the measurement of tracer concentration for this flow cell and for all capillary sizes.

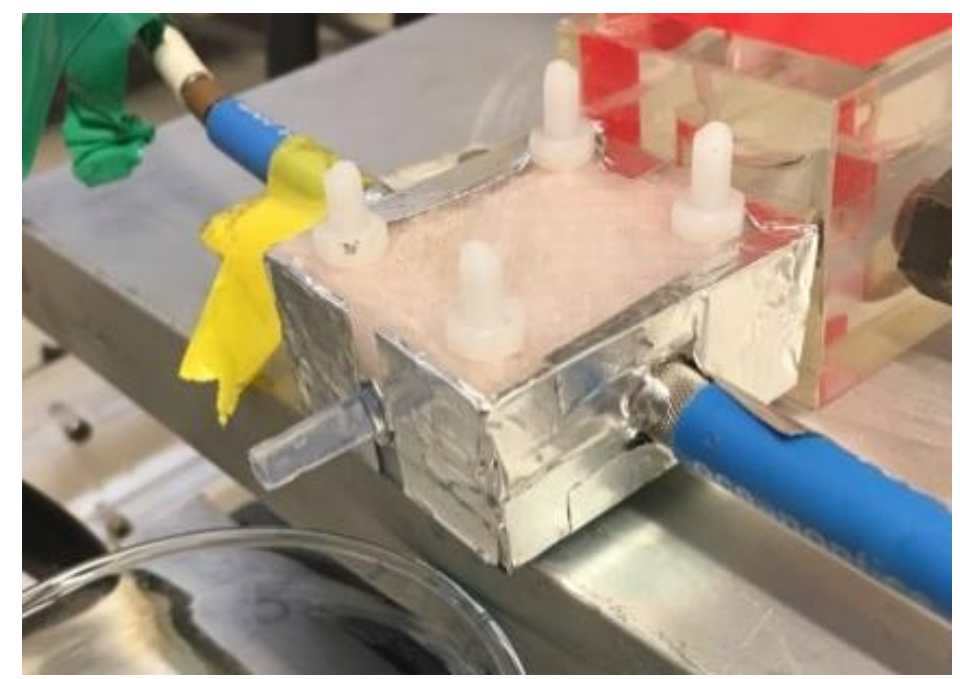

Figure S6: Custom made "Flow through cell" (as in fig. S5) for through the wall absorbance measurements. 


\section{Magnitude of the velocity inside each capillary and sonication residence time}

The velocity magnitude maps under the horn in the $3.2 \mathrm{~mm}$ I.D. capillary tube are presented in Figures S79. In Figure S9, we can observe that the velocity magnitude of the vortices is much larger than the velocity magnitude of the bulk flow, demonstrating that acoustic streaming dominates. On the contrary, Figure S7 shows only a slight deviation from the Poiseuille flow velocity profile, and acoustic streaming is negligible. In Figure S8, the onset of acoustic streaming occurs. The bulk flow velocity is still larger than the velocity inside the vortices. Nevertheless, in this case, we cannot neglect acoustic streaming (or assume a Poiseuille flow velocity profile). The velocity magnitude maps under the horn in the $1.55 \mathrm{~mm}$ I.D. capillary tube are presented in Figures S10-12. In every case, for the $1.55 \mathrm{~mm}$ I.D. capillary tube, the Poiseuille flow velocity profile can be assumed.

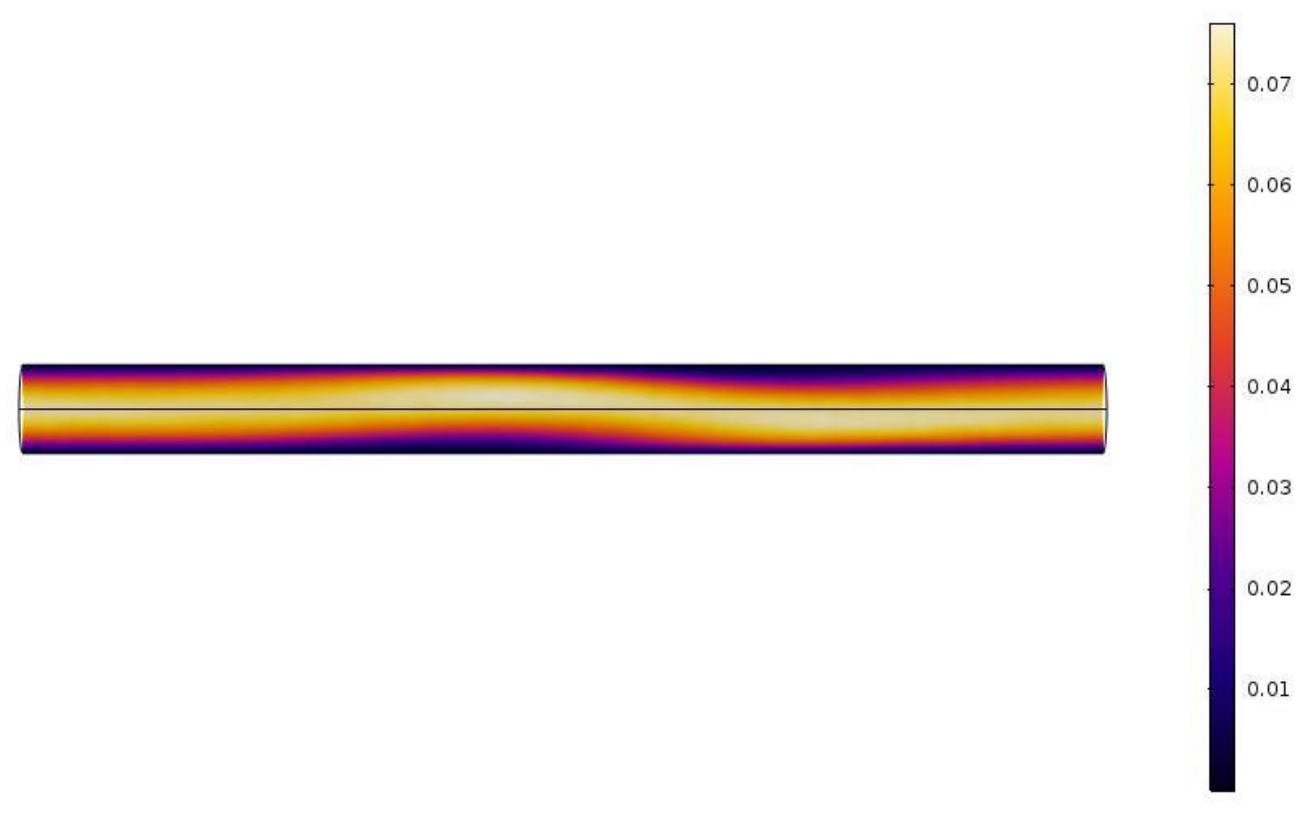

Figure S7: Velocity magnitude $(\mathrm{m} / \mathrm{s})$ inside the $3.2 \mathrm{~mm}$ I.D. capillary at sonication residence time of $0.3 \mathrm{~s}$.

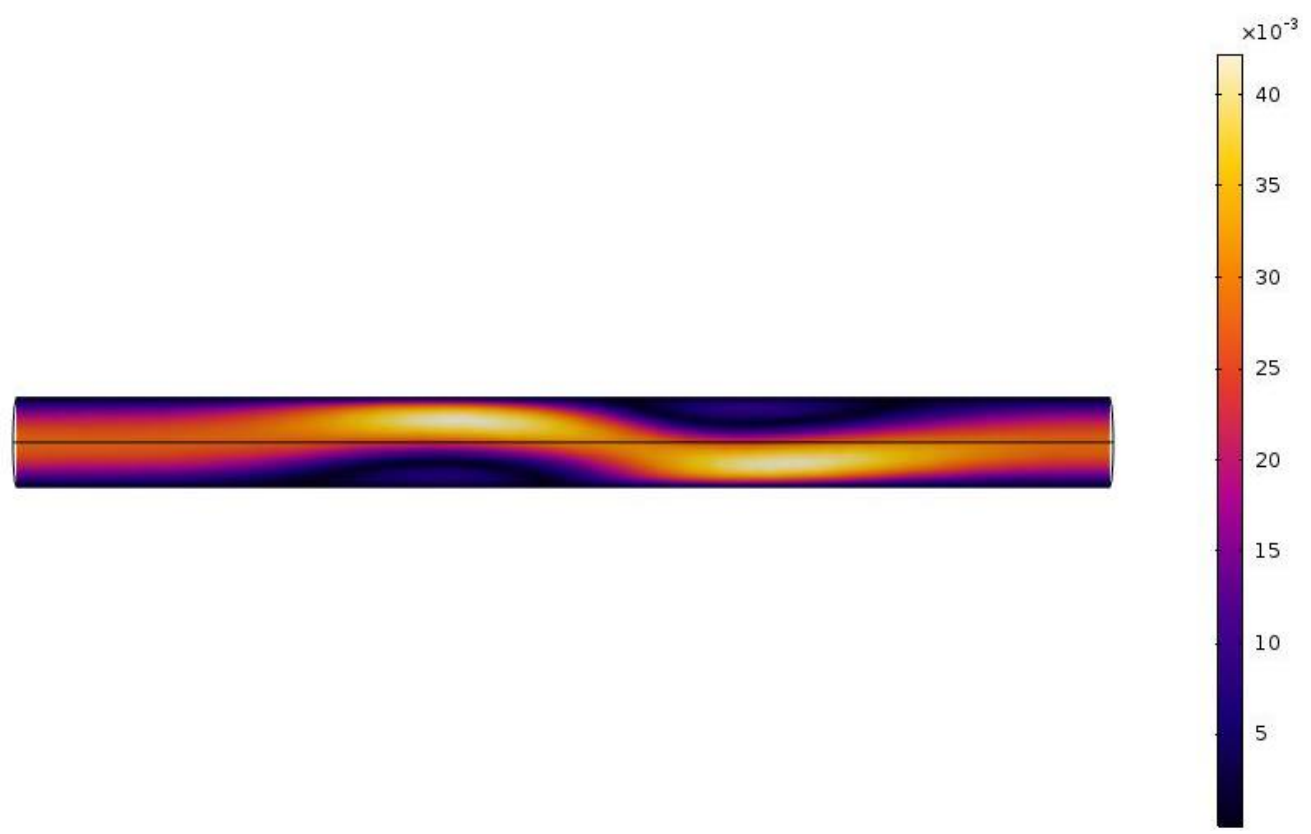

Figure S8: Velocity magnitude $(\mathrm{m} / \mathrm{s})$ inside $3.2 \mathrm{~mm}$ I.D. capillary at sonication residence time of $1 \mathrm{~s}$. 


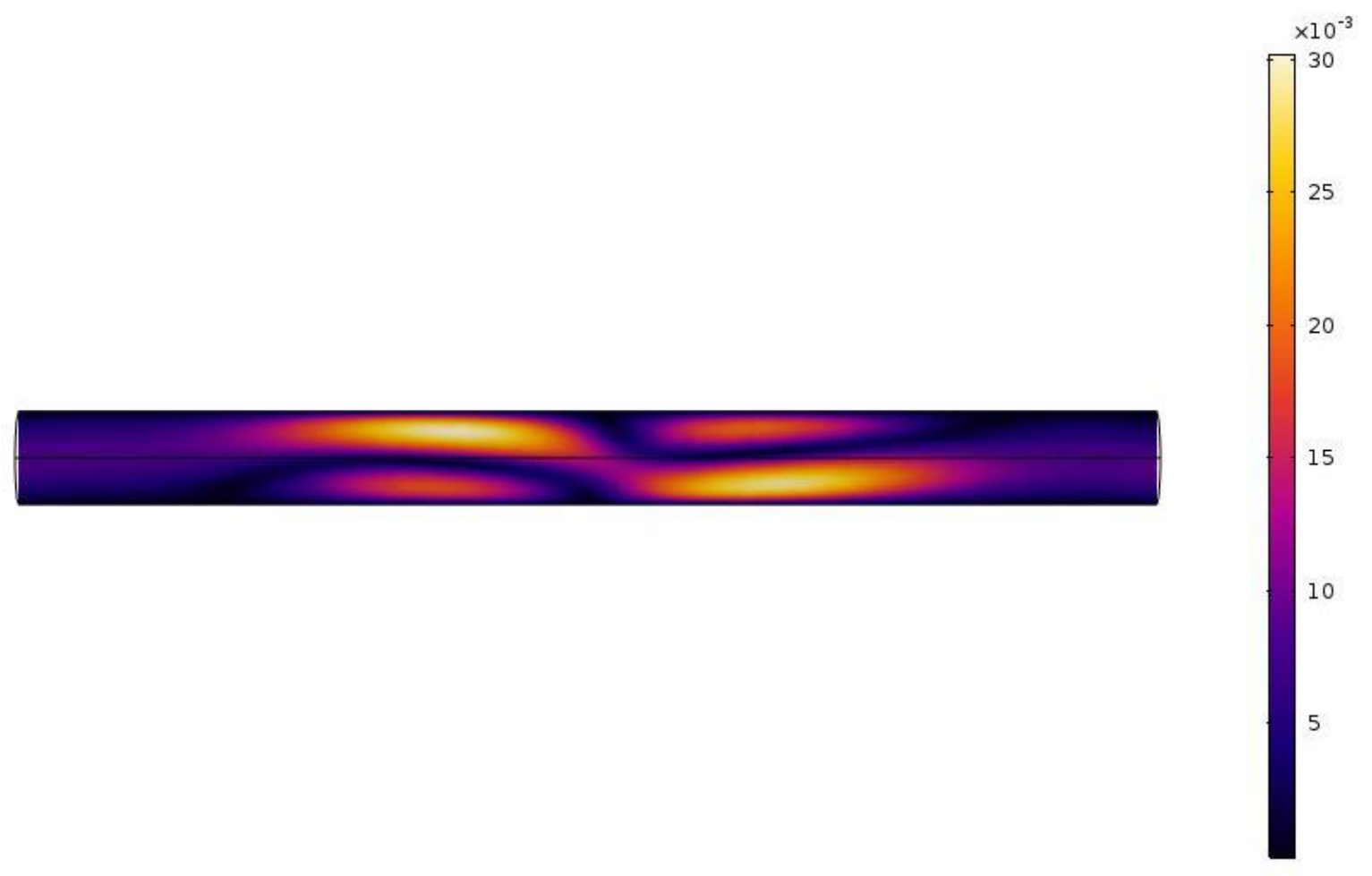

Figure S9: Velocity magnitude $(\mathrm{m} / \mathrm{s})$ inside $3.2 \mathrm{~mm}$ I.D. capillary at sonication residence time of $3 \mathrm{~s}$.

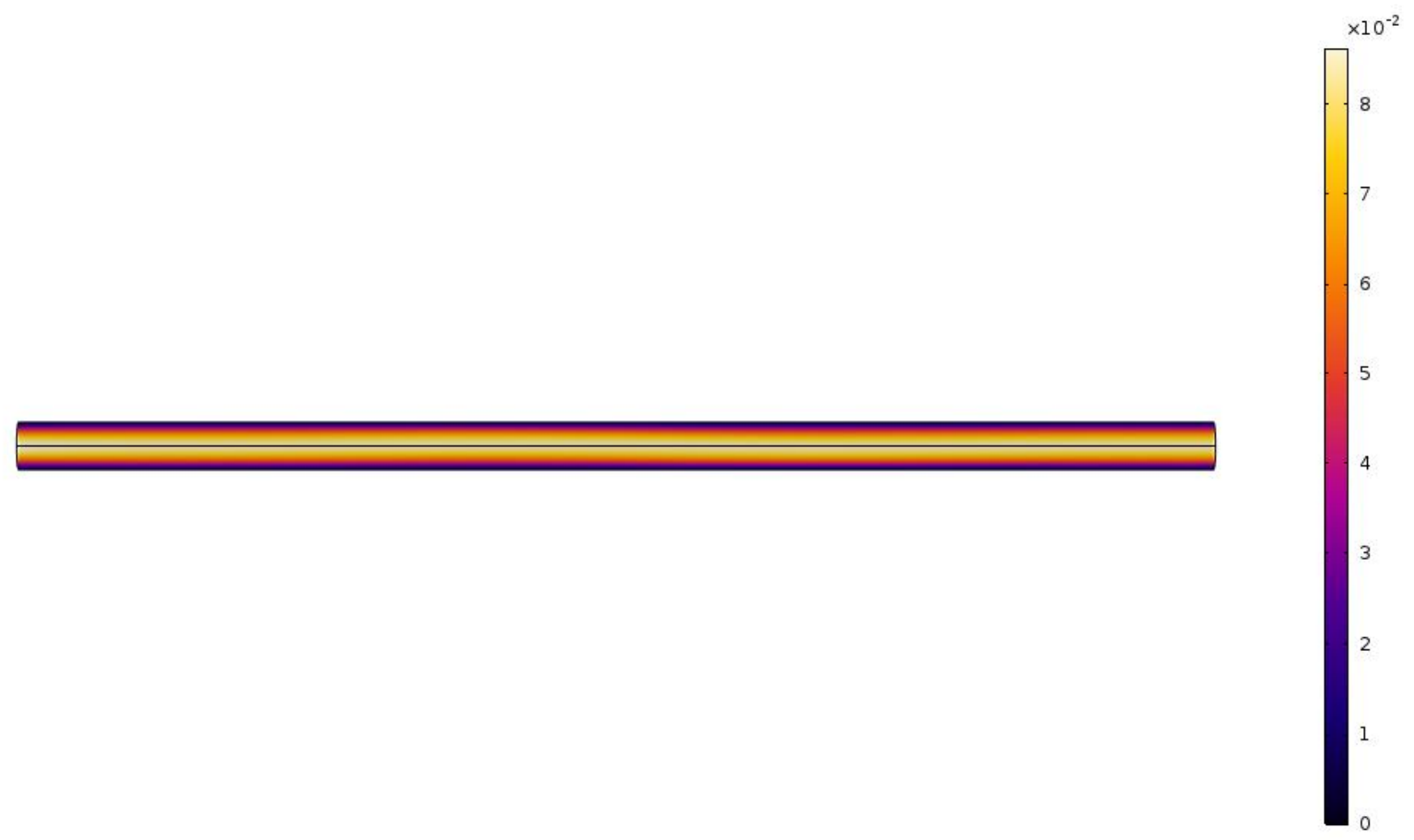

Figure S10: Velocity magnitude $(\mathrm{m} / \mathrm{s})$ inside $1.55 \mathrm{~mm}$ I.D. capillary at sonication residence time of $0.3 \mathrm{~s}$. 
Figure S11: Velocity magnitude $(\mathrm{m} / \mathrm{s})$ inside $1.55 \mathrm{~mm}$ I.D. capillary at sonication residence time of $1 \mathrm{~s}$.

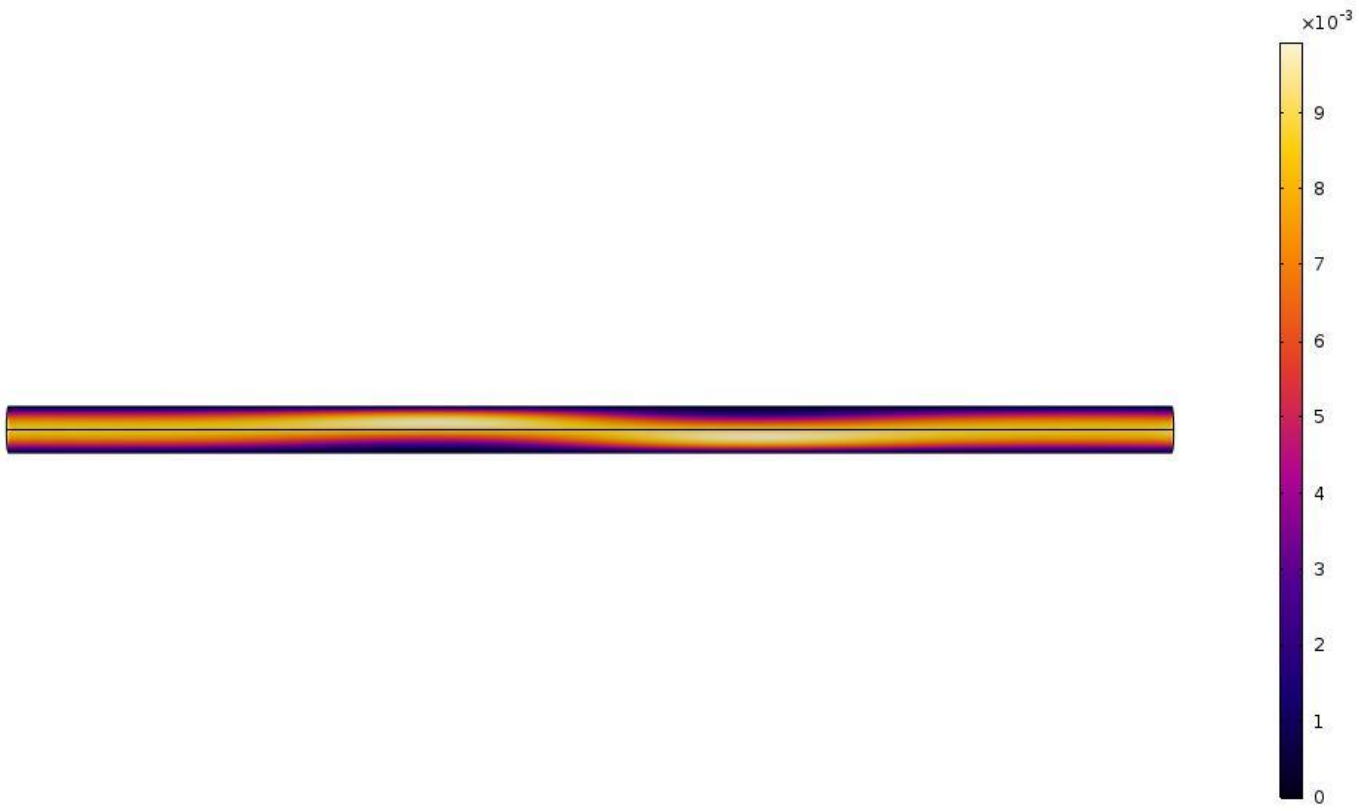

Figure S12: Velocity magnitude $(\mathrm{m} / \mathrm{s})$ inside $1.55 \mathrm{~mm}$ I.D. capillary at sonication residence time of $3 \mathrm{~s}$. 


\section{Statistical hypothesis testing}

We conducted a statistical hypothesis test between each averaged mean crystal size in Figure 12 . This test is used to determine if the means of two sets of data are significantly different from each other. Detailed explanation and uses of this test are found in Morris and Langari [5]. For such test, the t-value between two data points is obtained as follows:

$$
t=\frac{\overline{x_{1}}-\overline{x_{2}}}{\sqrt{\frac{\sigma_{1}}{n_{1}}+\frac{\sigma_{2}}{n_{2}}}}
$$

where $\bar{x}$ is the averaged mean size, $\sigma$ is the standard deviation (i.e. error bars in the Figures) and $n$ the number of data samples. This value is compared to the values in the distribution of critical $t$-values reported in the Handbook of Tables for Probability and Statistics [6]. A confidence level of $95 \%$ is commonly accepted in the literature and assumed here. Based on the comparison between the calculated $t$-value and the critical t-value in the literature, we either reject or accept the hypothesis. Such analysis is conducted 3 times to compare each mean crystal size to the other two. In every case, a significant difference between the two means is found. This shows that despite the partially overlapping error bars, the means are statistically different and hence there exists a trend. This quantitatively validates the decrease in mean crystal size that is observed in Figure 12.

We have also calculated the exact $p$-values for each comparison. The $p$-value is the probability that the hypothesis is incorrect. The critical p-value at which the hypothesis is rejected is 0.05 (dictated by the confidence level). Even in the worst case scenario, the probability that two data sets have the same mean is 0.02 , which is below the scientifically accepted threshold. The results are displayed in Table S1.

Table S1: Statistical hypothesis test for the presence of a trend in Figure R4.

\begin{tabular}{|l|c|c|c|}
\hline Hypothesis & $D_{\tau=0.3 s} \neq D_{\tau=1 s}$ & $D_{\tau=1 s} \neq D_{\tau=3 s}$ & $D_{\tau=0.3 s} \neq D_{\tau=3 s}$ \\
\hline $\mathrm{t}$-value & 2.164 & 3.017 & 3.867 \\
\hline Critical t-value & 1.729 & 1.725 & 1.753 \\
\hline p-value & 0.02078 & 0.00317 & 0.00048 \\
\hline
\end{tabular}

* $D$ - averaged mean crystal size

\section{Bibliography}

[1] K.W. Commander, A. Prosperetti, Linear pressure waves in bubbly liquids: Comparison Between Theory And Experiments, J. Acoust. Soc. Am. 85 (1989).

[2] O. Louisnard, A simple model of ultrasound propagation in a cavitating liquid. Part I: Theory, nonlinear attenuation and traveling wave generation, Ultrason. Sonochem. 19 (2012) 56-65.

[3] S. Lesnik, R. Mettin, G. Brenner, Study of ultrasound propagation and cavitation activity in a packing bed of spherical particles, Chemie Ing. Tech. 89 (2017) 1379-1384.

[4] G. Pfefer, R. Boistelle, Theoretical morphology of adipic acid crystals, J. Cryst. Growth. 208 (2000) 615-622.

[5] A.S. Morris, R.B.T. Langari, Measurement and instrumentation, Butterworth-Heinemann, Boston, (2012)

[6] W. H. Beyer, Handbook of Tables for Probability and Statistics (2nd ed.), CRC Press, Boca Raton, Florida, 1986 